\title{
Total Synthesis of Asperazine
}

\section{Steven P. Govek and Larry E. Overman*}

Department of Chemistry, 516 Rowland Hall, University of California, Irvine, CA 92697-2025

\section{Supporting Information ${ }^{1,2}$}

Experimental procedures and characterization data for key transformations (preparation of 8, 9, 12, 14, and $\mathbf{1}$ ) copies of ${ }^{1} \mathrm{H}$ and ${ }^{13} \mathrm{C}$ NMR spectra of these compounds, tables comparing NMR spectra of synthetic and natural $\mathbf{1}$, and the CD spectrum of synthetic 1 (24 pages).

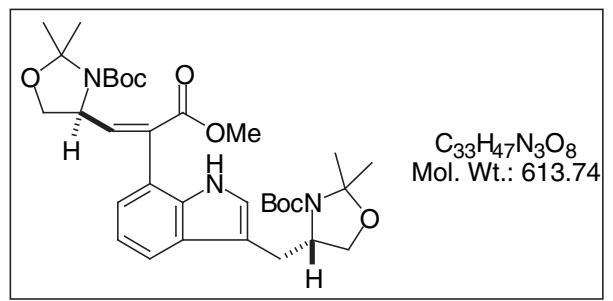

Stille Coupling to Prepare 8 and Deprotection to Form Enoate 9.

A suspension of $\mathrm{Pd}_{2}(\mathrm{dba})_{3} \cdot \mathrm{CHCl}_{3}(145 \mathrm{mg}, 0.280 \mathrm{mmol} \mathrm{Pd})$, (2-furyl $)_{3} \mathrm{P}(265 \mathrm{mg}, 1.14 \mathrm{mmol})$, and dry NMP $(8 \mathrm{~mL})$ in a basewashed, flame-dried $100 \mathrm{~mL}$ Schlenk flask was stirred for $2 \mathrm{~h}$ to furnish a yellow-green homogenous solution. A solution of stannane $5(1.70 \mathrm{~g}, 2.96 \mathrm{mmol})$, iodide $7(1.04 \mathrm{~g}, 1.87 \mathrm{mmol})$, and NMP $(12 \mathrm{~mL})$ was added, and the reaction was degassed by the freeze-pump-thaw method at $-78{ }^{\circ} \mathrm{C}$ three times. Copper(I) iodide $(620 \mathrm{mg}$, $3.26 \mathrm{mmol}$ ) was added, the mixture was stirred for $19 \mathrm{~h}$ at $\mathrm{rt}$, and the reaction was quenched by slow addition of saturated aqueous KF $(10 \mathrm{~mL})$. After stirring for $15 \mathrm{~min}$, the mixture was diluted with MTBE $(400 \mathrm{~mL})$ and washed with water $(300 \mathrm{~mL})$ containing concentrated ammonia solution $(15 \mathrm{~mL})$. The aqueous layer was extracted with MTBE $(200 \mathrm{~mL})$. The combined organic extracts were dried $\left(\mathrm{MgSO}_{4}\right)$, filtered, concentrated, and the residue was purified by silica gel chromatography (13:1, 9:1, 6:1, 4:1 hexanes-ethyl acetate) to give $1.19 \mathrm{~g}$ of 8 as a colorless foam. Diagnostic data: MS (ESI) $\mathrm{m} / z 736.43$ (736.38 calcd for $\mathrm{C}_{38} \mathrm{H}_{55} \mathrm{~N}_{3} \mathrm{NaO}_{10}, \mathrm{M}+\mathrm{Na}$ ).

A solution of $8(1.19 \mathrm{~g})$ and DMSO (25 mL) was degassed [bubbling argon (30 min), evacuate (15 min, $5 \mathrm{~mm}$ ), bubbling argon $(30 \mathrm{~min})]$ and then heated at $130{ }^{\circ} \mathrm{C}$ for $6.5 \mathrm{~h}$. The reaction was poured into water (200 $\mathrm{mL})$ and extracted with $\mathrm{Et}_{2} \mathrm{O}(50 \mathrm{~mL} \times 3)$. The combined organic extracts were dried $\left(\mathrm{MgSO}_{4}\right)$, filtered, concentrated, and the residue was purified by silica gel chromatography (8:1, 6:1, 5:1 hexanes-ethyl acetate) to give $939 \mathrm{mg}(82 \%)$ of 9 as a colorless foam: $[\alpha]^{24}{ }_{\mathrm{D}}=-41.7,[\alpha]^{24}{ }_{577}=-43.9,[\alpha]^{24}{ }_{546}=-52.6,[\alpha]^{24}{ }_{435}=-126$, $[\alpha]^{24}{ }_{405}=-166\left(\mathrm{c} 0.79, \mathrm{CHCl}_{3}\right) ;{ }^{1} \mathrm{H}$ NMR $\left(500 \mathrm{MHz}, \mathrm{DMSO}, 80^{\circ} \mathrm{C}\right) \delta 10.23(\mathrm{~s}, 1 \mathrm{H}, \mathrm{NH}), 7.61(\mathrm{br} \mathrm{d}, J=7.7 \mathrm{~Hz}$, $1 \mathrm{H}, \operatorname{Ar} H), 7.13(\mathrm{~d}, J=2.3 \mathrm{~Hz}, 1 \mathrm{H}, \operatorname{Ar} H), 7.01(\mathrm{t}, J=7.6 \mathrm{~Hz}, 1 \mathrm{H}, \operatorname{Ar} H), 6.92(\mathrm{dd}, J=7.2,0.8 \mathrm{~Hz}, 1 \mathrm{H}, \operatorname{Ar} H)$, $6.28(\mathrm{~d}, J=8.0 \mathrm{~Hz}, 1 \mathrm{H}, \mathrm{C}=\mathrm{CH}), 5.16($ app td, $J=7.4,4.3 \mathrm{~Hz}, 1 \mathrm{H}, \mathrm{CHN}), 4.29\left(\mathrm{dd}, J=9.0,7.0 \mathrm{~Hz}, 1 \mathrm{H}, \mathrm{CH}_{2} \mathrm{O}\right)$, 4.14-4.10 (m, $1 \mathrm{H}, \mathrm{CHN}), 3.98\left(\mathrm{br}, 1 \mathrm{H}, \mathrm{CH}_{2} \mathrm{O}\right), 3.80\left(\mathrm{dd}, J=7.9,5.8 \mathrm{~Hz}, 1 \mathrm{H}, \mathrm{CH}_{2} \mathrm{O}\right), 3.74(\mathrm{dd}, J=8.8,1.6 \mathrm{~Hz}$, $1 \mathrm{H}, \mathrm{CH}_{2} \mathrm{O}$ ), 3.68 (s, $3 \mathrm{H}, \mathrm{CO}_{2} \mathrm{Me}$ ), 3.15 (dd, $\left.J=13.8,2.8 \mathrm{~Hz}, 1 \mathrm{H}, \mathrm{CH}_{2}\right), 2.81\left(\mathrm{dd}, J=13.8,10.2 \mathrm{~Hz}, 1 \mathrm{H}, \mathrm{CH}_{2}\right.$ ), $1.53\left(\mathrm{~s}, 6 \mathrm{H}, \mathrm{Me}_{2} \mathrm{C}\right), 1.50(\mathrm{~s}, 3 \mathrm{H}, \mathrm{MeCMe}), 1.48(\mathrm{~s}, 9 \mathrm{H}, t$-Bu), 1.44 (s, 9H, $t$-Bu), 1.43 (s, $3 \mathrm{H}, \mathrm{MeCMe}) ;{ }^{13} \mathrm{C}$ NMR $\left(125 \mathrm{MHz}\right.$, DMSO, $\left.80^{\circ} \mathrm{C}\right) \delta 165.8,151.2,151.0,146.7,134.0,130.2,127.5,123.4,121.7,121.3,118.0$,

\footnotetext{
${ }^{1}$ General experimental details have been described: Minor, K. P.; Overman, L. E. J. Org. Chem. 1997, 62, 6379-6387.

${ }^{2}$ Abbreviations not defined in J. Org. Chem. 2001, 66, 24A: dba = trans, trans-dibenzylideneacetone, NMP = 1-methyl-2pyrrolidinone, $\mathrm{SEMCl}=2$-(trimethylsilyl)ethoxymethyl chloride, $\mathrm{DMA}=N, N$-dimethylacetamide, $\mathrm{HATU}=O$-(7-azabenzotriazol-1yl)- $N, N, N$ ', $N$ '-tetramethyluronium hexafluorophosphate (Carpino, L. A. J. Am. Chem. Soc. 1993, 115, 4397-4398), MTBE = methyl tert-butyl ether.
} 
117.9, 110.9, 93.3, 92.8, 79.2, 78.7, 67.6, 65.8, 56.9, 56.1, 51.2, 28.4, 27.8, 27.7, 26.7, 25.9, 24.1, 23.4; IR (neat) 3317, 2979, 1698, 1667, 1392, $1366 \mathrm{~cm}^{-1}$; MS (FAB) $\mathrm{m} / \mathrm{z} 613.3363$ (613.3363 calcd for $\mathrm{C}_{33} \mathrm{H}_{47} \mathrm{~N}_{3} \mathrm{O}_{8}, \mathrm{M}$ ). Anal. Calcd for $\mathrm{C}_{33} \mathrm{H}_{47} \mathrm{~N}_{3} \mathrm{O}_{8}$ : C, 64.58; H, 7.72; N, 6.85. Found: C, 64.44; H, 7.91; N, 6.75.

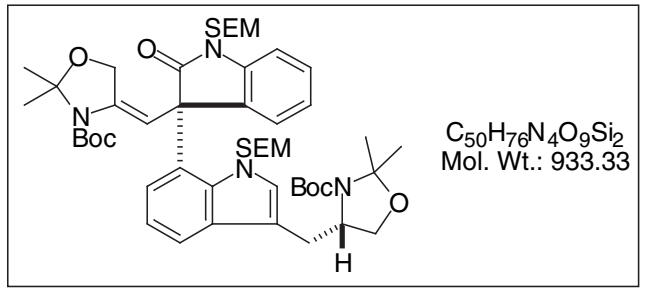

Heck Cyclization to Form Oxindole 12. A suspension of $\mathrm{Pd}_{2}(\mathrm{dba})_{3} \cdot \mathrm{CHCl}_{3}(195 \mathrm{mg}, 0.377 \mathrm{mmol}$ $\left.\mathrm{Pd}^{0}\right)$, (2-furyl $)_{3} \mathrm{P}(440 \mathrm{mg}, 1.90 \mathrm{mmol})$, and dry DMA $(15 \mathrm{~mL})$ in a base-washed, flame-dried $100 \mathrm{~mL}$ Schlenk flask was stirred for $2 \mathrm{~h}$ to furnish a yellow-green homogenous solution. Anilide $\mathbf{1 1}(2.00 \mathrm{~g}, 1.88 \mathrm{mmol})$, 1,2,2,6,6-pentamethylpiperidine $(2.00 \mathrm{~mL}, 11.1 \mathrm{mmol})$, and DMA $(5 \mathrm{~mL})$ were added, and the reaction was degassed by the freeze-pump-thaw method at $-78{ }^{\circ} \mathrm{C}$ three times. After $10 \mathrm{~h}$ at $90{ }^{\circ} \mathrm{C}$, the reaction was allowed to cool to rt, poured into saturated aqueous $\mathrm{LiCl}(100 \mathrm{~mL})$, diluted with water $(100 \mathrm{~mL})$, and extracted with MTBE $(100 \mathrm{~mL} \times 3)$. The combined organic extracts were dried $\left(\mathrm{MgSO}_{4}\right)$, filtered, concentrated, and the residue was purified by silica gel chromatography (12:1, 9:1, 6:1, 4:1 hexanes-ethyl acetate) to give $1.16 \mathrm{~g}$ $(66 \%)$ of 12 as a colorless foam: $[\alpha]_{D}^{27}=-120,[\alpha]_{577}^{27}=-127,[\alpha]_{546}^{27}=-147,[\alpha]_{435}^{27}=-305,[\alpha]_{405}^{27}=-409(\mathrm{c}$ $\left.0.75, \mathrm{CHCl}_{3}\right) ;{ }^{1} \mathrm{H}$ NMR $\left(500 \mathrm{MHz}, \mathrm{DMSO}, 100{ }^{\circ} \mathrm{C}\right) \delta 7.62(\mathrm{~d}, J=7.4 \mathrm{~Hz}, 1 \mathrm{H}, \operatorname{Ar} H), 7.36(\mathrm{td}, J=7.7,1.2 \mathrm{~Hz}$, $1 \mathrm{H}, \operatorname{Ar} H), 7.28(\mathrm{~s}, 1 \mathrm{H}, \operatorname{Ar} H), 7.21(\mathrm{~d}, J=7.0 \mathrm{~Hz}, 1 \mathrm{H}, \operatorname{Ar} H), 7.19(\mathrm{~d}, J=7.7 \mathrm{~Hz}, 1 \mathrm{H}, \operatorname{Ar} H), 7.10(\mathrm{td}, J=7.3,0.9$ $\mathrm{Hz}, 1 \mathrm{H}, \mathrm{Ar} H), 6.93(\mathrm{t}, J=7.7 \mathrm{~Hz}, 1 \mathrm{H}, \mathrm{Ar} H), 6.76(\mathrm{br} \mathrm{d}, J=7.1 \mathrm{~Hz}, 1 \mathrm{H}, \mathrm{Ar} H), 6.41$ (t, $J=1.9 \mathrm{~Hz}, 1 \mathrm{H}, \mathrm{C}=\mathrm{CH})$, $5.71\left(\mathrm{br}, 2 \mathrm{H}, \mathrm{NCH}_{2} \mathrm{O}\right), 5.19\left(\mathrm{~d}, J=10.9 \mathrm{~Hz}, 1 \mathrm{H}, \mathrm{NCH}_{2} \mathrm{O}\right), 5.14\left(\mathrm{~d}, J=10.9 \mathrm{~Hz}, 1 \mathrm{H}, \mathrm{NCH}_{2} \mathrm{O}\right), 4.16-4.10(\mathrm{~m}$, $1 \mathrm{H}, \mathrm{CHN}), 3.83\left(\mathrm{dd}, J=8.2,6.0 \mathrm{~Hz}, 1 \mathrm{H}, \mathrm{CH}_{2} \mathrm{O}\right), 3.77\left(\mathrm{dd}, J=12.9,1.8 \mathrm{~Hz}, 1 \mathrm{H}, \mathrm{CH}_{2} \mathrm{O}\right), 3.73(\mathrm{dd}, J=8.7,1.9$ $\left.\mathrm{Hz}, 1 \mathrm{H}, \mathrm{CH}_{2} \mathrm{O}\right), 3.72\left(\mathrm{dd}, J=13.5,1.8 \mathrm{~Hz}, 1 \mathrm{H}, \mathrm{CH}_{2} \mathrm{O}\right), 3.62-3.54\left(\mathrm{~m}, 2 \mathrm{H}, \mathrm{CH}_{2} \mathrm{O}\right), 3.46-3.39\left(\mathrm{br} \mathrm{m}, 1 \mathrm{H}, \mathrm{CH}_{2} \mathrm{O}\right)$, 3.34-3.27 (br m, $1 \mathrm{H}, \mathrm{CH}_{2} \mathrm{O}$ ), 3.16 (dd, $J=14.0,3.2 \mathrm{~Hz}, 1 \mathrm{H}, \mathrm{CH}_{2}$ ), 2.84 (dd, $J=14.0,10.0 \mathrm{~Hz}, 1 \mathrm{H}, \mathrm{CH}_{2}$ ), 1.51 (s, $3 \mathrm{H}, \mathrm{MeCMe}$ ), 1.50 (s, 9H, $t$-Bu), 1.45 (s, 3H, MeCMe), 1.44 (s, 3H, MeCMe), 1.43 (s, 9H, $t$-Bu), 1.41 (s, 3H, $\mathrm{MeCMe}$ ), 0.92-0.75 (m, 4H, $\mathrm{CH}_{2} \mathrm{Si}$ ), -0.04 (s, 9H $\mathrm{Me}_{3} \mathrm{Si}$ ), -0.07 (s, 9H Me $\mathrm{Mi}_{3} \mathrm{Si} ;{ }^{13} \mathrm{C}$ NMR (125 MHz, DMSO, $\left.100{ }^{\circ} \mathrm{C}\right) \delta 177.1,150.9,149.6,140.6,136.8,134.0,133.6,131.3,127.9(2), 124.5,123.6,122.83,122.75,118.6$, 118.3, 112.3, 109.2, 105.5, 95.1, 92.7, 80.9, 78.7, 78.0, 69.0, 65.7, 65.1, 64.2, 63.6, 56.9, 56,7, 27.7 (2), 27.6, 26.9, 24.7, 24.4, 23.8, 17,1, 17,0, -2.06, -2.12; IR (neat) 2953, 1703, 1384, 1366, $1074 \mathrm{~cm}^{-1}$; MS (ESI) $\mathrm{m} / \mathrm{z}$ 955.5060 (955.5049 calcd for $\mathrm{C}_{50} \mathrm{H}_{76} \mathrm{~N}_{4} \mathrm{NaO}_{9} \mathrm{Si}_{2}, \mathrm{M}+\mathrm{Na}$ ). Anal. Calcd for $\mathrm{C}_{50} \mathrm{H}_{76} \mathrm{~N}_{4} \mathrm{O}_{9} \mathrm{Si}_{2}: \mathrm{C}, 64.34 ; \mathrm{H}, 8.21 ; \mathrm{N}$, 6.00. Found: C, 64.64; H, 8.27; N, 5.93.

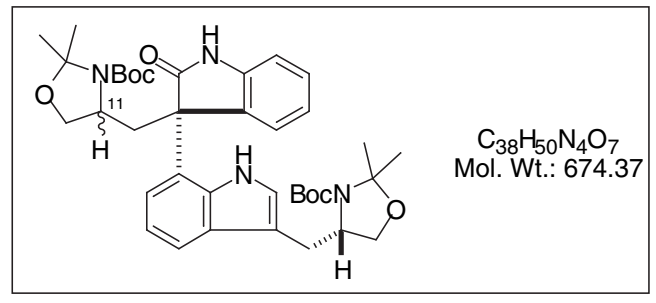

Hydrogenation of 13 to Form 14. A mixture of $\mathbf{1 3}$ (400 mg, $0.595 \mathrm{mmol}$ ), palladium on carbon (158 $\mathrm{mg}, 10 \mathrm{wt} \%, 0.148 \mathrm{mmol})$ and DMF $(12 \mathrm{~mL})$ was stirred in a pressure reactor under $1000 \mathrm{psi}$ of hydrogen for $24 \mathrm{~h}$ at rt. This mixture was filtered through Celite, and the resulting filter cake was washed with MTBE (300 $\mathrm{mL})$. The filtrate was washed with saturated aqueous $\mathrm{LiCl}(100 \mathrm{~mL})$, and the resulting aqueous layer was extracted with MTBE $(100 \mathrm{~mL})$. The combined organic extracts were dried $\left(\mathrm{MgSO}_{4}\right)$, filtered, concentrated, and 
the residue was purified by silica gel chromatography $(4: 1,3: 1$ hexanes-ethyl acetate) to give $383 \mathrm{mg}(95 \%)$ of 14, a colorless foam, as an inseparable 4:1 mixture of epimers. All characterization data was obtained on this mixture: IR (neat) 3335, 2978, 1698, 1389, $1366 \mathrm{~cm}^{-1}$; MS (CI) $\mathrm{m} / z 674.3667$ (674.3680 calcd for $\mathrm{C}_{38} \mathrm{H}_{50} \mathrm{~N}_{4} \mathrm{O}_{7}$, M). Anal. Calcd for $\mathrm{C}_{38} \mathrm{H}_{50} \mathrm{~N}_{4} \mathrm{O}_{7}$ : C, 67.63; H, 7.47; N, 8.30. Found: C, 67.48; H, 7.51; N, 8.08.

NMR data for the major epimer $(\mathrm{C} 11 \alpha-\mathrm{H}):{ }^{3} \mathrm{H}$ NMR $\left(500 \mathrm{MHz}, \mathrm{DMSO}, 100{ }^{\circ} \mathrm{C}\right) \delta 10.43(\mathrm{~s}, 1 \mathrm{H}, \mathrm{NH})$, 9.87 (s, 1H, NH), 7.59-7.54 (m, 1H, ArH), 7.39 (d, J=7.4 Hz, 1H, ArH), 7.32 (td, J = 7.7, 1.2 Hz, 1H, ArH), $7.17(\mathrm{~d}, J=2.4 \mathrm{~Hz}, 1 \mathrm{H}, \operatorname{ArH}), 7.12(\mathrm{td}, J=7.5,1.0 \mathrm{~Hz}, 1 \mathrm{H}, \operatorname{Ar} H), 7.01(\mathrm{~d}, J=7.5 \mathrm{~Hz}, 1 \mathrm{H}, \operatorname{ArH}), 6.97-6.91(\mathrm{~m}$, $2 \mathrm{H}, \mathrm{ArH}), 4.15-4.08(\mathrm{~m}, 1 \mathrm{H}, \mathrm{CHN}), 3.83\left(\mathrm{dd}, J=8.0,5.9 \mathrm{~Hz}, 1 \mathrm{H}, \mathrm{CH} \mathrm{H}_{2} \mathrm{O}\right) 3.77(\mathrm{dd}, J=8.7,1.8 \mathrm{~Hz}, 1 \mathrm{H}$, $\left.\mathrm{CH}_{2} \mathrm{O}\right), 3.73\left(\mathrm{dd}, \mathrm{J}=8.8,1.9 \mathrm{~Hz}, 1 \mathrm{H}, \mathrm{CH}_{2} \mathrm{O}\right), 3.65-3.54\left(\mathrm{~m}, 2 \mathrm{H}, \mathrm{CH}_{2} \mathrm{O}\right.$ and $\left.\mathrm{CHN}\right), 3.14(\mathrm{dd}, J=14.0,3.6 \mathrm{~Hz}$, $\left.1 \mathrm{H}, \mathrm{CH}_{2}\right), 2.93\left(\mathrm{dd}, J=13.6,3.2 \mathrm{~Hz}, 1 \mathrm{H}, \mathrm{CH}_{2}\right), 2.80\left(\mathrm{dd}, J=14.1,4.3 \mathrm{~Hz}, 1 \mathrm{H}, \mathrm{CH}_{2}\right), 2.61(\mathrm{~d}, J=13.1 \mathrm{~Hz}, 1 \mathrm{H}$, $\left.\mathrm{CH}_{2}\right), 1.51$ (s, 3H, MeCMe), 1.50 (s, 3H, MeCMe), 1.46 (s, 9H, $t$-Bu), 1.44 (s, 3H, MeCMe), $1.37(\mathrm{~s}, 9 \mathrm{H}, t-\mathrm{Bu})$, 1.35 (s, 3H, MeCMe); ${ }^{13} \mathrm{C}$ NMR (125 MHz, DMSO, $\left.100{ }^{\circ} \mathrm{C}\right) \delta 179.5,150.9,150.5,140.9,133.5,129.3,128.9$, $127.9,126.0,123.3,123.0,121.0,119.0,118.0,117.6,110.9,109.7,92.6,91.8,78.8,78.5,65.9,65.1,56.9$, 54.9, 54.0, 37.0, 28.1, 27.6, 27.5, 26.5, 26.4, 23.5, 23.4.

NMR data for the minor epimer $(\mathrm{C} 11 \beta-\mathrm{H}):{ }^{3,4}{ }^{1} \mathrm{H}$ NMR $\left(500 \mathrm{MHz}, \mathrm{DMSO}, 100{ }^{\circ} \mathrm{C}\right) \delta 10.36(\mathrm{~s}, 1 \mathrm{H}, \mathrm{NH})$, $10.08(\mathrm{~s}, 1 \mathrm{H}, \mathrm{NH}), 7.57(1 \mathrm{H}, \operatorname{ArH}), 7.41(\mathrm{~d}, J=7.6 \mathrm{~Hz}, 1 \mathrm{H}, \operatorname{ArH}), 7.32(1 \mathrm{H}, \operatorname{ArH}), 7.17(1 \mathrm{H}, \operatorname{ArH}), 7.12(1 \mathrm{H}$, $\mathrm{ArH}), 7.02-6.91(3 \mathrm{H}, \mathrm{ArH}), 4.12(1 \mathrm{H}), 4.04-3.98(\mathrm{~m}, 1 \mathrm{H}, \mathrm{CHN}), 3.83(1 \mathrm{H}), 3.72(\mathrm{dd}, J=8.8,2.0 \mathrm{~Hz}, 1 \mathrm{H}$, $\left.\mathrm{CH}_{2} \mathrm{O}\right), 3.29\left(\mathrm{dd}, \mathrm{J}=8.8,5.8 \mathrm{~Hz}, 1 \mathrm{H}, \mathrm{CH}_{2} \mathrm{O}\right), 3.15\left(1 \mathrm{H}, \mathrm{CH}_{2} \mathrm{O}\right), 2.99\left(\mathrm{~d}, J=14.0 \mathrm{~Hz}, 1 \mathrm{H}, \mathrm{CH}_{2}\right), 2.86-2.77(2 \mathrm{H})$, $2.49\left(1 \mathrm{H}, \mathrm{CH}_{2}\right), 1.47$ (s, 9H, $t$-Bu), 1.45 (s, 3H, MeCMe), 1.43 (s, 9H, $t$-Bu), 1.42 (s, 3H, MeCMe), 1.39 (s, 3H, $\mathrm{MeCMe}$ ), 1.33 (s, 3H, MeCMe); ${ }^{13} \mathrm{C}$ NMR (125 MHz, DMSO, $100{ }^{\circ} \mathrm{C}$, diagnostic signals only) $\delta 178.5,150.7$, $141.1,133.7,130.3,128.7,127.9,125.8,123.0,122.2,121.0,119.5,117.7,111.0,109.8,91.7,78.9,65.8,65.0$, $54.8,54.2,37.4,28.0,26.4,26.2,23.4$.

Asperazine 1. A solution of $17(21.7 \mathrm{mg}, 0.0211 \mathrm{mmol})$ and formic acid (6 mL) was maintained at $\mathrm{rt}$ for $2 \mathrm{~h}$. The formic acid was then removed as an azeotrope with heptane $(15 \mathrm{~mL} \times 3)$. Diagnostic data for the product: MS (ESI) $m / z 729.42$ (729.34 calcd for $\mathrm{C}_{42} \mathrm{H}_{45} \mathrm{~N}_{6} \mathrm{O}_{6}, \mathrm{M}+\mathrm{H}$ ).

A solution of this deprotected tetrapeptide, acetic acid $(400 \mu \mathrm{L}, 7.0 \mathrm{mmol})$, and $n$-butanol $(9.6 \mathrm{~mL})$ was refluxed for $24 \mathrm{~h}$. The acetic acid and $n$-butanol were then removed in vacuo $(0.5 \mathrm{~mm})$, and the resulting residue was purified by preparative TLC (9:1 chloroform-methanol) to give $8.2 \mathrm{mg}(59 \%)$ of asperazine (1) as a colorless solid. This material showed ${ }^{1} \mathrm{H}$ NMR spectra in $\mathrm{CDCl}_{3}, \mathrm{CH}_{2} \mathrm{Cl}_{2}$ and $\mathrm{CD}_{3} \mathrm{CN},{ }^{13} \mathrm{C}$ NMR spectra in $\mathrm{CDCl}_{3}$ and $\mathrm{CD}_{3} \mathrm{CN}$, mass spectral data, and a $\mathrm{CD}$ spectrum in $\mathrm{MeOH}$ that compared favorably to those of the natural isolate. ${ }^{5}$ The optical rotation of synthetic asperazine, $[\alpha]_{\mathrm{D}}+95.7\left(\mathrm{c} 0.2, \mathrm{CH}_{3} \mathrm{OH}\right)$ was higher than that reported for the natural material, $[\alpha]_{\mathrm{D}}+52\left(\mathrm{c} 0.2, \mathrm{CH}_{3} \mathrm{OH}\right){ }^{5}$

\footnotetext{
${ }^{3}$ The stereochemistry of the major and minor hydrogenation products was established by ${ }^{1} \mathrm{H}$ NOE analysis of $\mathbf{1 6}$.

${ }^{4}$ In cases where the multiplicity and/or peak assignment was not made, the peak was obstructed by the major isomer.

${ }^{5}$ (a) Varoglu, M.; Corbett, T. H.; Valeriote, F. A.; Crews, P. J. Org. Chem. 1997, 62, 7078-7079. (b) Unpublished data for natural 1 kindly provided by Professor Phillip Crews.
} 
Table 1: ${ }^{1} \mathrm{H}$ NMR Data (500 MHz, $\mathrm{CD}_{3} \mathrm{CN}$ ) for Natural and Synthetic Asperazine. ${ }^{6}$

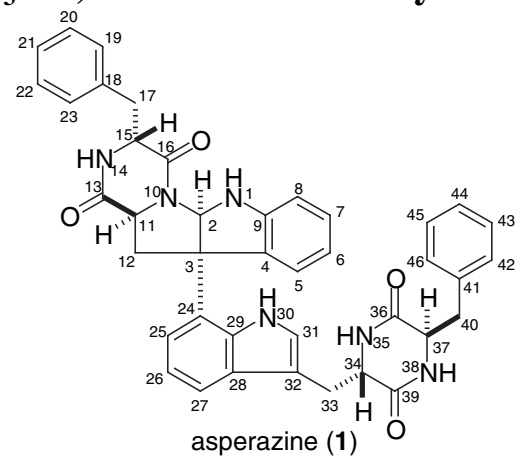

\begin{tabular}{|c|c|c|}
\hline Position & Natural & Synthetic \\
\hline 30 & 8.61 (br s) & 8.82 (br s) \\
\hline 27 & $7.48(\mathrm{dd}, J=7,1 \mathrm{~Hz})$ & $7.49(\mathrm{ddd}, J=7.6,1.3,0.7 \mathrm{~Hz})$ \\
\hline $42,44,46$ & $7.27(\mathrm{~m})$ & $7.30-7.23(\mathrm{~m})$ \\
\hline 21 & $7.20(\mathrm{~m})$ & $7.23-7.18(\mathrm{~m})$ \\
\hline $\begin{array}{l}19,20,22,23,25,26 \\
43,45\end{array}$ & $7.12(\mathrm{~m})$ & $7.14-7.06(\mathrm{~m})$ \\
\hline 31 & $6.918(\mathrm{~d}, J=2.5 \mathrm{~Hz})$ & $6.915(\mathrm{~d}, J=2.6 \mathrm{~Hz})$ \\
\hline 5 & $6.82(\mathrm{dd}, J=7,1 \mathrm{~Hz})$ & $6.815(\mathrm{ddd}, J=7.9,1.0,0.6 \mathrm{~Hz})$ \\
\hline 8 & $6.815(\mathrm{~d}, J=7.5 \mathrm{~Hz})$ & $6.80(\mathrm{ddd}, J=7.8,0.6,0.6 \mathrm{~Hz})$ \\
\hline 6 & $6.66(\mathrm{dd}, J=7,1 \mathrm{~Hz})$ & $6.66(\mathrm{ddd}, J=7.5,7.5,1.0 \mathrm{~Hz})$ \\
\hline 38 & $6.49(\mathrm{~d}, J=4.5 \mathrm{~Hz})$ & $6.55(\mathrm{br} \mathrm{d}, J=3.9 \mathrm{~Hz})$ \\
\hline 14 & $6.18(\mathrm{br} \mathrm{s})$ & $6.26(\mathrm{br} \mathrm{d}, J=0.8 \mathrm{~Hz})$ \\
\hline 35 & $6.16(\mathrm{br} \mathrm{s})$ & $6.23(\mathrm{br} \mathrm{d}, J=0.5 \mathrm{~Hz})$ \\
\hline 1 & 6.14 (br s) & $6.16(\mathrm{br} \mathrm{d}, J=2.2 \mathrm{~Hz})$ \\
\hline 2 & $5.71(\mathrm{~d}, J=1 \mathrm{~Hz})$ & $5.71(\mathrm{~d}, J=2.3 \mathrm{~Hz})$ \\
\hline 37 & $4.12(\mathrm{ddd}, J=6,5,4.5 \mathrm{~Hz})$ & $4.12(\mathrm{dddd}, J=6.3,4.4,4.4,0.5 \mathrm{~Hz})$ \\
\hline 15 & $3.59(\mathrm{ddd}, J=5.5,5,4.5 \mathrm{~Hz})$ & $3.59(\mathrm{dddd}, J=5.6,4.8,2.0,0.8 \mathrm{~Hz})$ \\
\hline 34 & $3.51(\mathrm{ddd}, J=6,5.5,3.5 \mathrm{~Hz})$ & $3.53(\mathrm{dddd}, J=5.7,4.7,1.8,0.8 \mathrm{~Hz})$ \\
\hline 11 & $3.32(\mathrm{dd}, J=9.5,7.5 \mathrm{~Hz})$ & $3.34(\mathrm{dd}, J=9.7,7.5 \mathrm{~Hz})$ \\
\hline 12 & $3.25(\mathrm{dd}, J=14,7.5 \mathrm{~Hz})$ & $3.23(\mathrm{dd}, J=13.3,7.5 \mathrm{~Hz})$ \\
\hline 33 & $3.09(\mathrm{dd}, J=14.5,5.5 \mathrm{~Hz})$ & $3.11(\mathrm{ddd}, J=14.9,5.8,0.7 \mathrm{~Hz})$ \\
\hline 40 & $3.06(\mathrm{dd}, J=14,6 \mathrm{~Hz})$ & $3.075(\mathrm{dd}, J=13.6,6.3 \mathrm{~Hz})$ \\
\hline 33 & $3.05(\mathrm{dd}, J=14.5,3.5 \mathrm{~Hz})$ & $3.070(\mathrm{ddd}, J=14.9,4.7,0.7 \mathrm{~Hz})$ \\
\hline 17 & $3.01(\mathrm{dd}, J=13.5,5.5 \mathrm{~Hz})$ & $3.00(\mathrm{dd}, J=13.9,5.6 \mathrm{~Hz})$ \\
\hline 17 & $2.95(\mathrm{dd}, J=13.5,5 \mathrm{~Hz})$ & $2.92(\mathrm{dd}, J=14.0,4.7 \mathrm{~Hz})^{7}$ \\
\hline 40 & $2.93(\mathrm{dd}, J=14,5 \mathrm{~Hz})$ & $2.96(\mathrm{dd}, J=13.7,4.9 \mathrm{~Hz})^{7}$ \\
\hline 12 & $2.40(\mathrm{dd}, J=14,9.5 \mathrm{~Hz})$ & $2.44(\mathrm{dd}, J=13.5,9.7 \mathrm{~Hz})$ \\
\hline
\end{tabular}

${ }^{6}$ Our assignments were made in analogy to those made by $\mathrm{Crews}^{5}$ unless otherwise noted.

${ }^{7} \mathrm{~A}{ }^{1} \mathrm{H}-{ }^{1} \mathrm{H}$ COSY experiment of synthetic 1 revealed this alternate assignment. 
Table 2: ${ }^{13} \mathrm{C}$ NMR Data $\left(125 \mathrm{MHz}, \mathrm{CD}_{3} \mathrm{CN}\right)$ for Natural and Synthetic Asperazine. 6

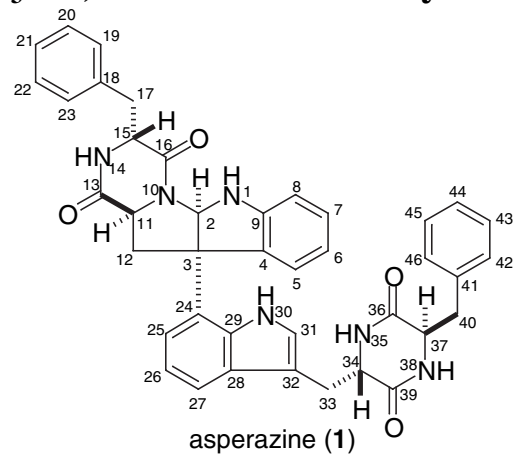

\begin{tabular}{|c|c|c|}
\hline Position & Natural & Synthetic \\
\hline 13 & 169.98 & 169.84 \\
\hline 36 & 169.64 & 169.43 \\
\hline 16 & 168.89 & 168.78 \\
\hline 39 & 168.84 & 168.67 \\
\hline 9 & 148.57 & 148.48 \\
\hline $18,41^{8}$ & 137.72 & 137.69 \\
\hline 29 & 135.45 & 135.42 \\
\hline 4 & 134.16 & 134.41 \\
\hline 42,46 & 131.63 & 131.60 \\
\hline 19,23 & 131.46 & 131.44 \\
\hline $7,28^{9}$ & 130.45 & $130.47,130.43$ \\
\hline 43,45 & 130.15 & 130.17 \\
\hline 20,22 & 130.09 & 130.11 \\
\hline 21 & 128.98 & 128.99 \\
\hline 44 & 128.72 & 128.70 \\
\hline $24^{10}$ & 126.31 & 126.57 \\
\hline $5,31^{11}$ & 125.20 & $126.28,125.21$ \\
\hline 6 & 120.93 & 121.50 \\
\hline 26 & 120.61 & 120.97 \\
\hline 25 & 120.24 & 120.63 \\
\hline 27 & 119.02 & 120.29 \\
\hline 8 & 112.41 & 112.41 \\
\hline 32 & 111.15 & 111.20 \\
\hline 2 & 84.20 & 84.46 \\
\hline 37 & 60.46 & 60.71 \\
\hline 3 & 59.28 & 59.38 \\
\hline 11 & 57.79 & 58.16 \\
\hline 15 & 57.14 & 57.40 \\
\hline 34 & 56.36 & 56.31 \\
\hline 40 & 40.72 & 41.18 \\
\hline 17 & 40.40 & 40.73 \\
\hline 12 & 39.55 & 40.01 \\
\hline 33 & 30.20 & 30.68 \\
\hline
\end{tabular}

\footnotetext{
${ }^{8}$ Non-equivalent overlapping carbons.

${ }^{9}$ Spectra of natural 1 show a single peak with a small shoulder. ${ }^{5}$ Our spectra show this peak resolved into two distinct peaks.

${ }^{10}$ Unpublished ${ }^{13} \mathrm{C}$ NMR spectra of natural 1 show a peak at $126.56 .{ }^{5}$ This peak was not assigned, presumably because it is barely larger than the noise. Our ${ }^{13} \mathrm{C}$ NMR spectra show this peak to be real and we assign it to C24.

${ }^{11}$ We assign the peak previously assigned to C24 (126.28), to either C5 or C31.
} 
CD Spectrum of Asperazine $(0.18 \mathrm{mg} / \mathrm{mL}$ methanol, $2 \mathrm{~mm}$ pathlength)

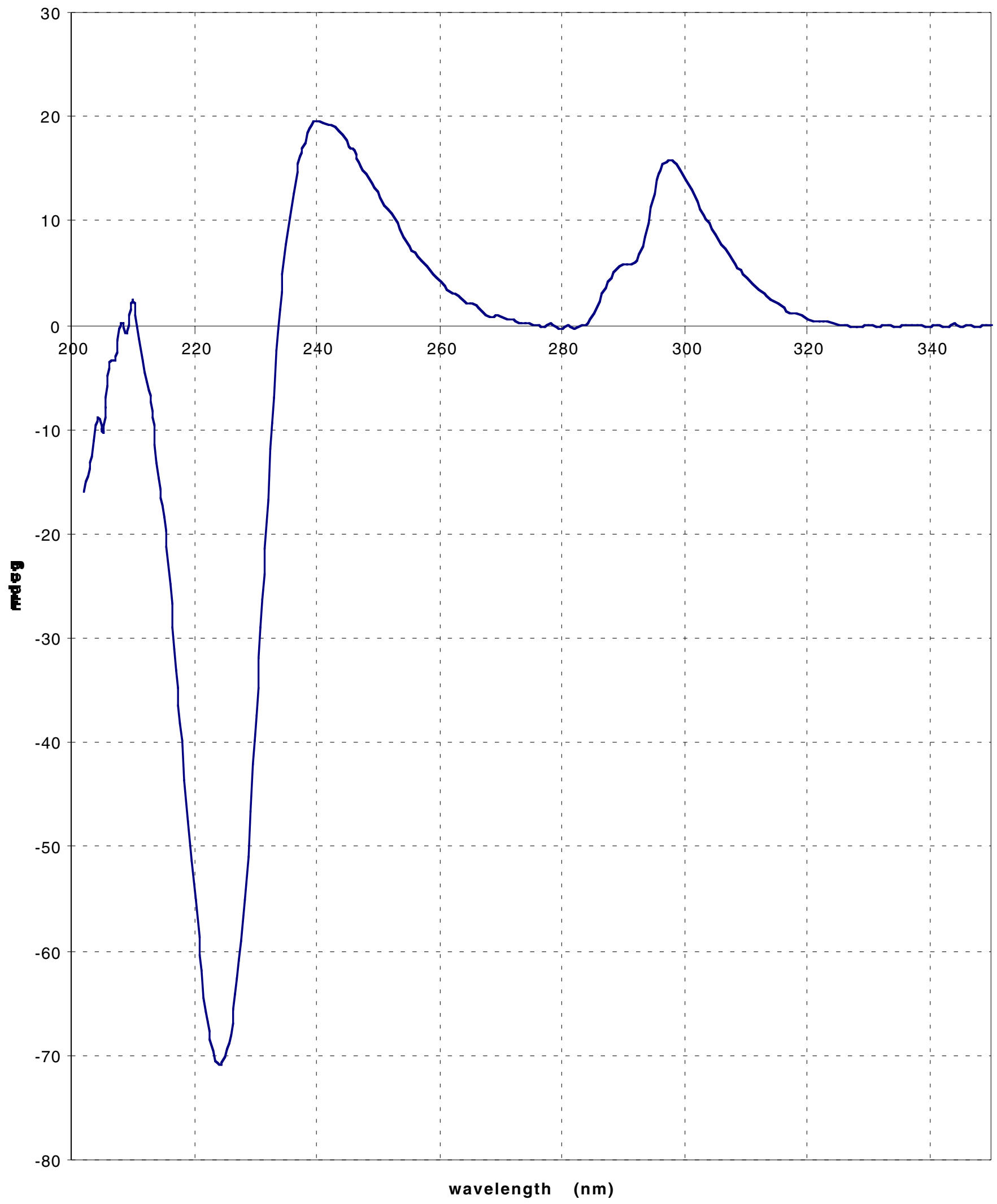



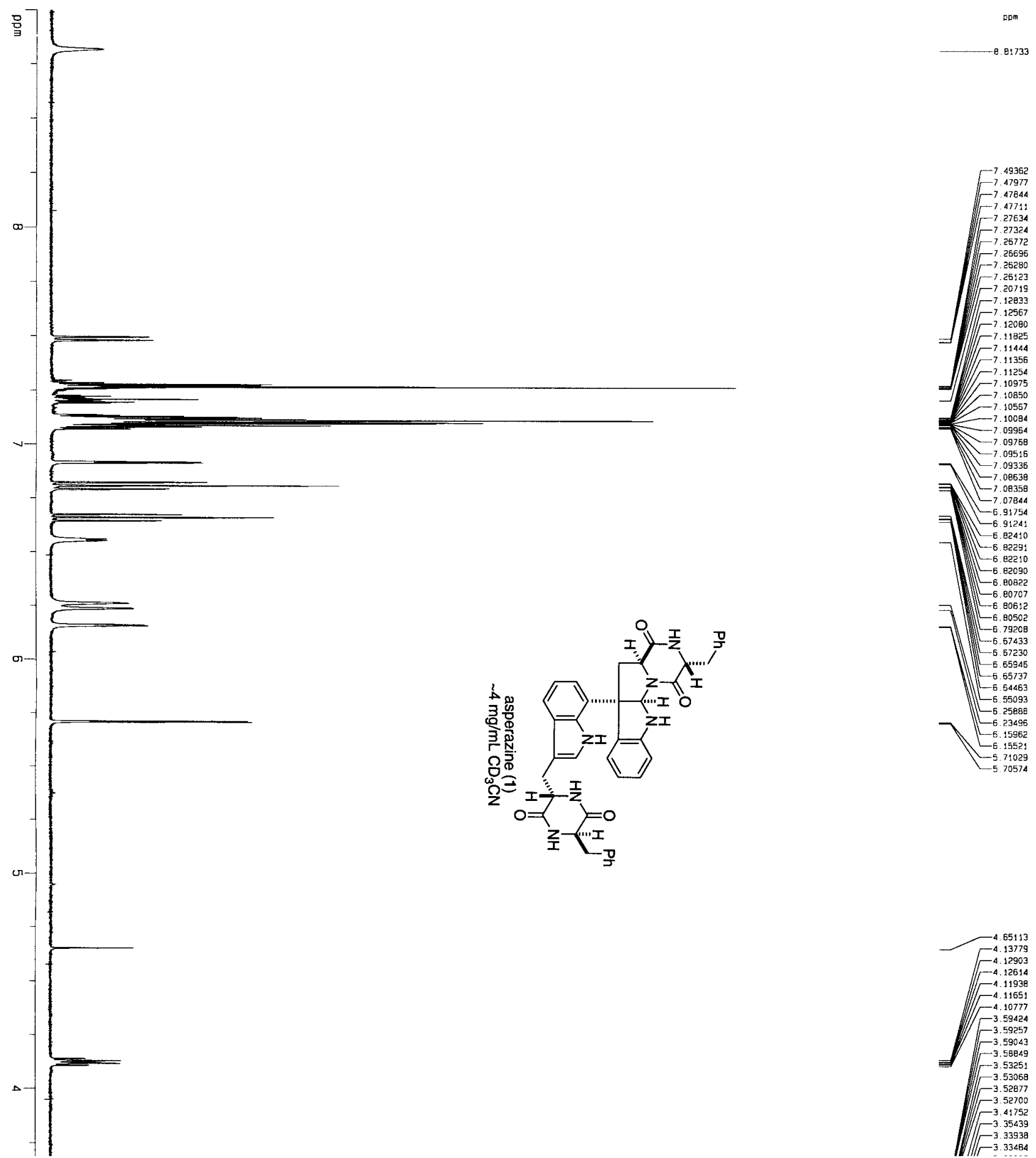


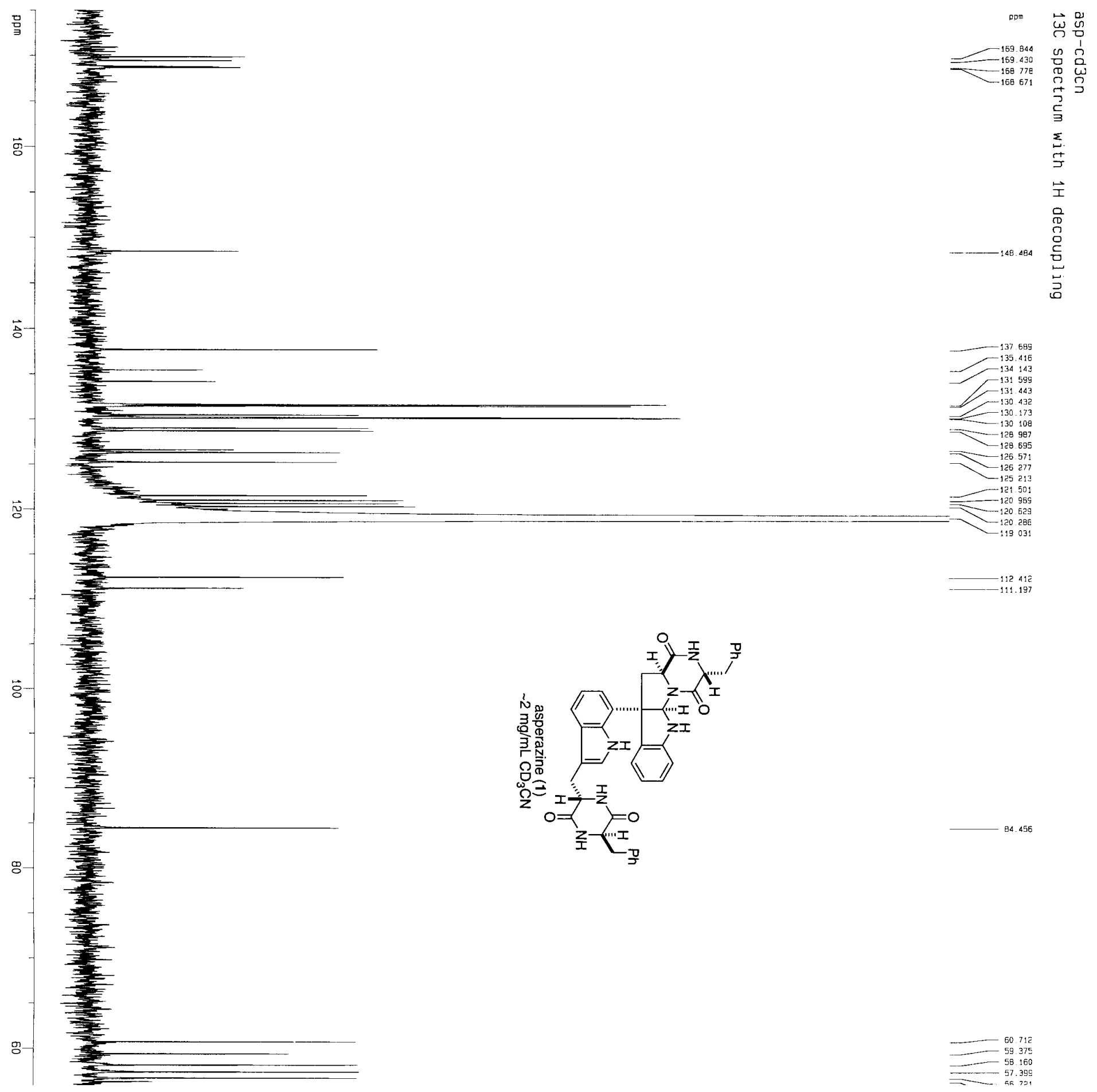




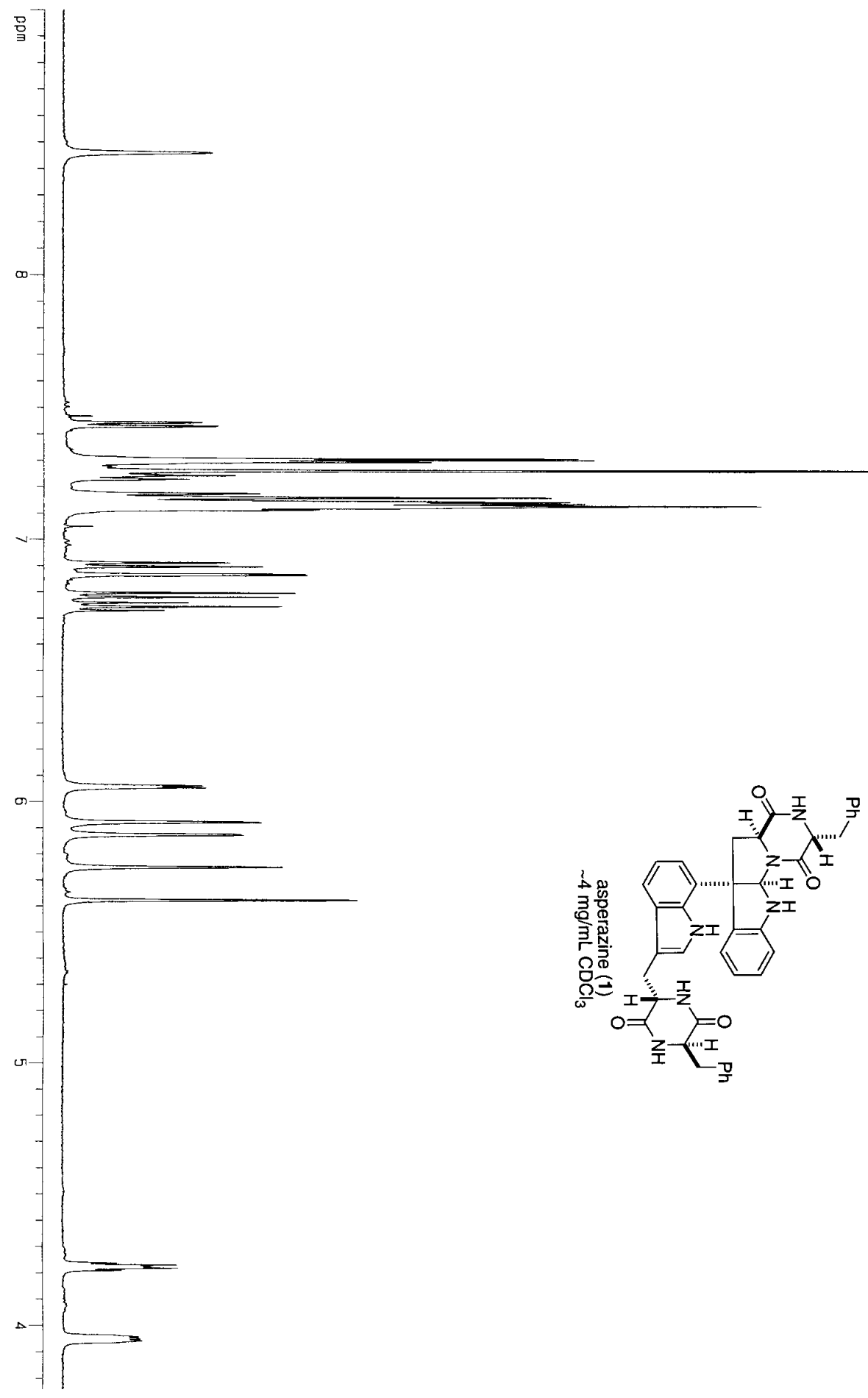

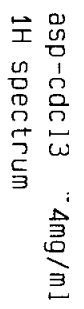
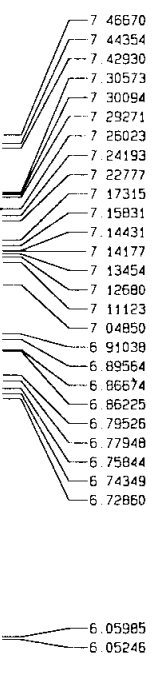

-591974
$-\square$
$-\quad 57122$

$-574853$

$-5.62295$

m

i




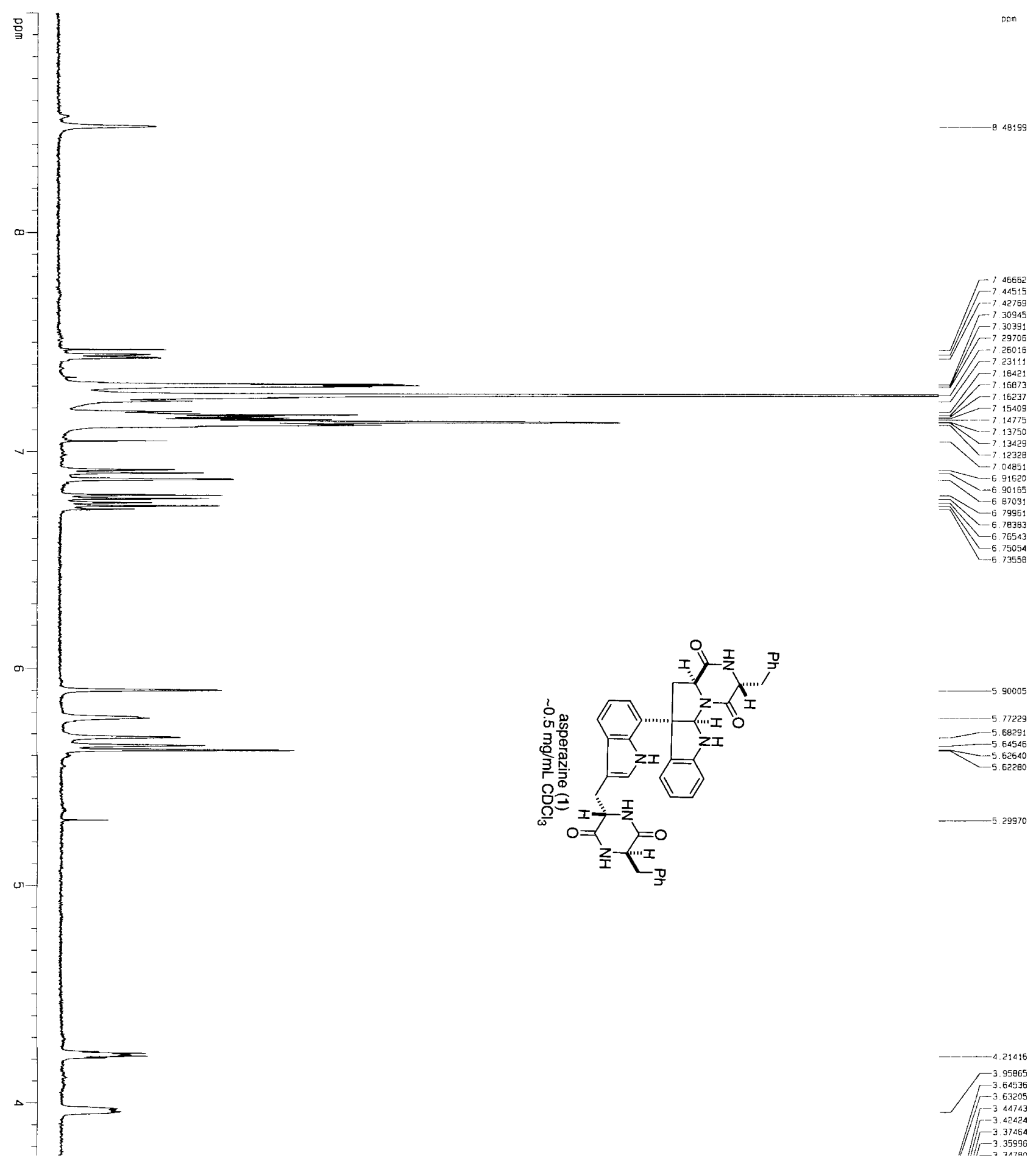




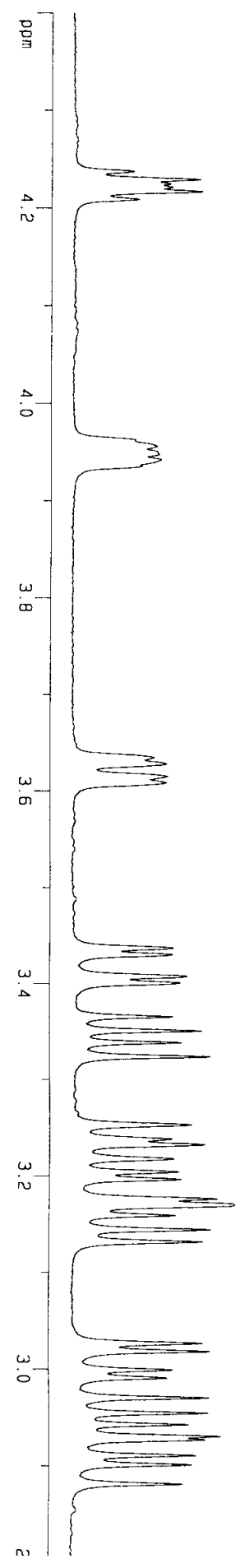

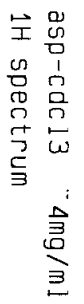
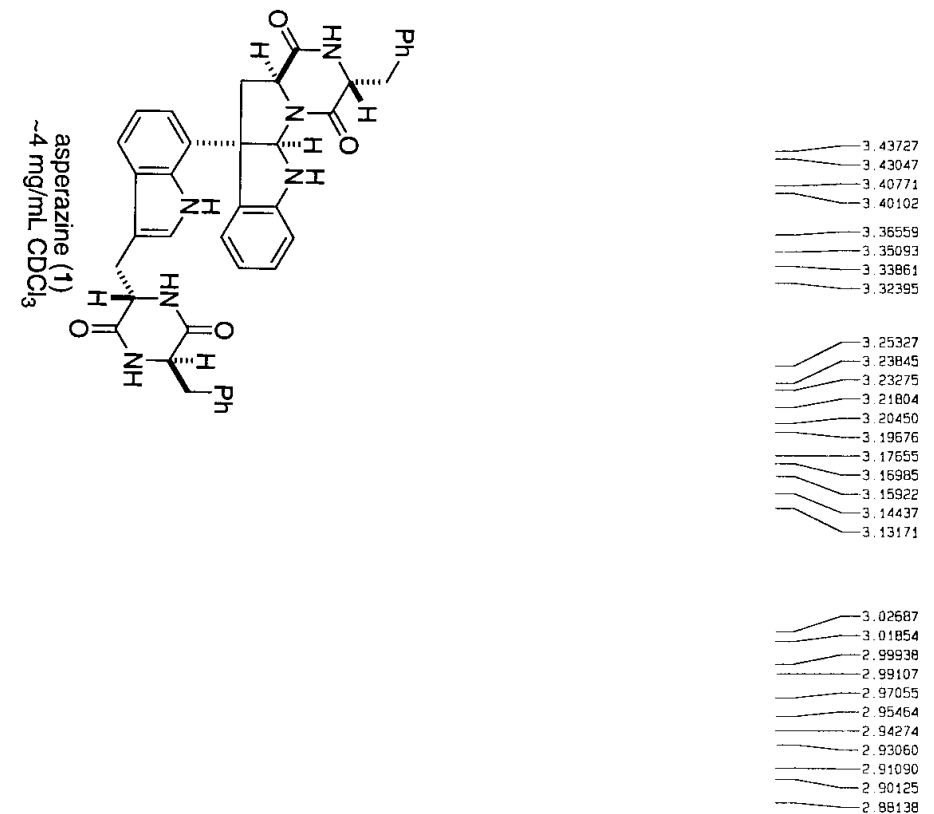

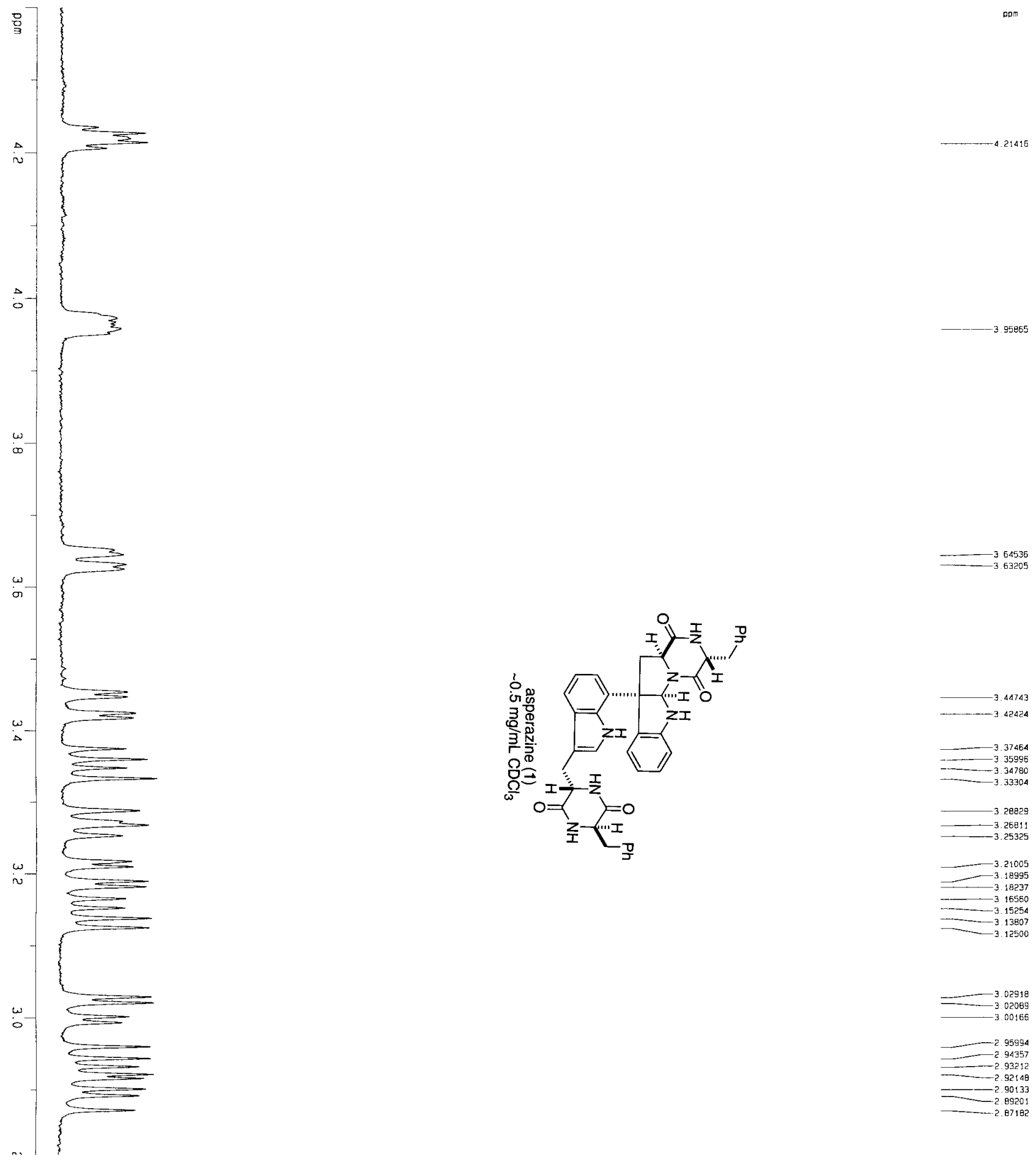


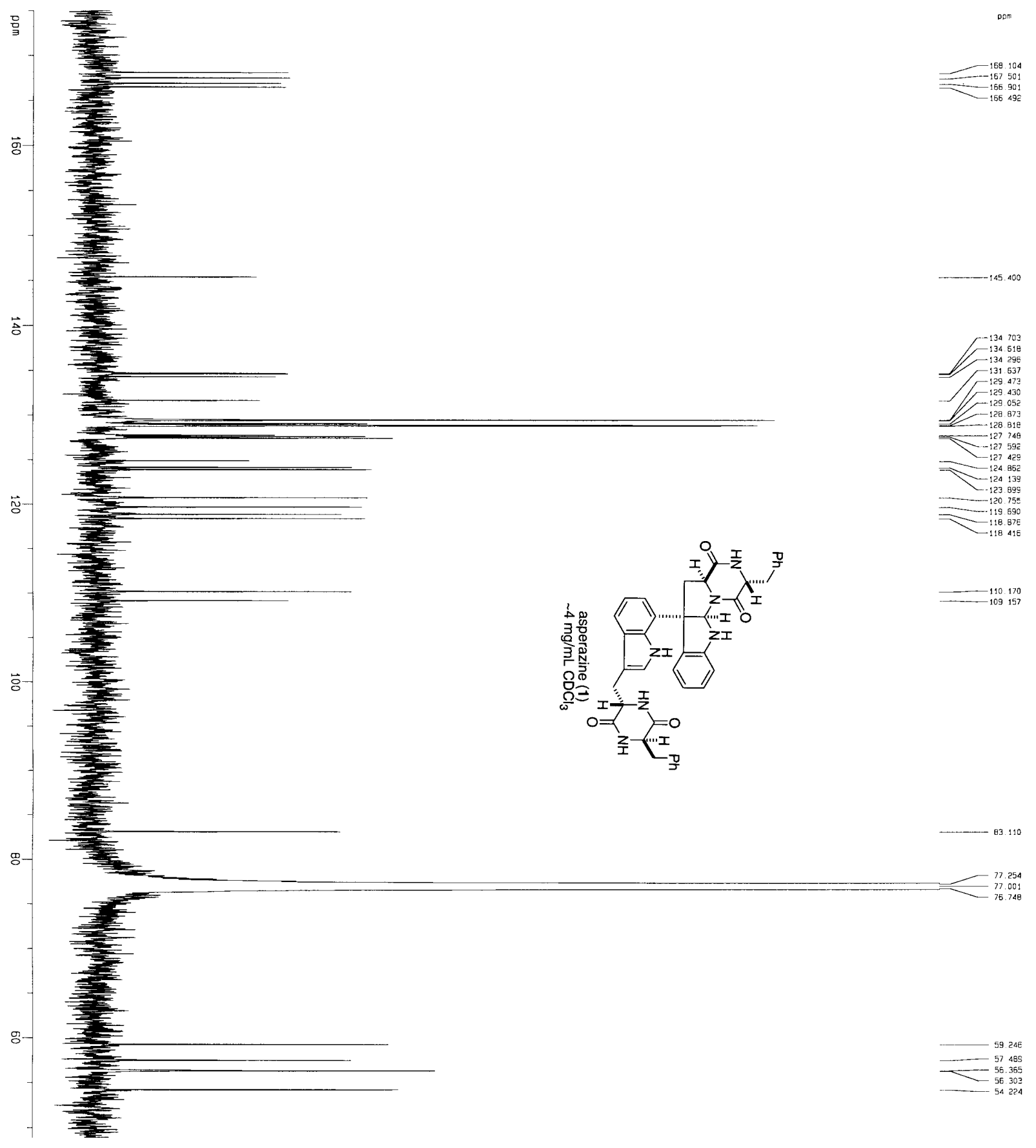




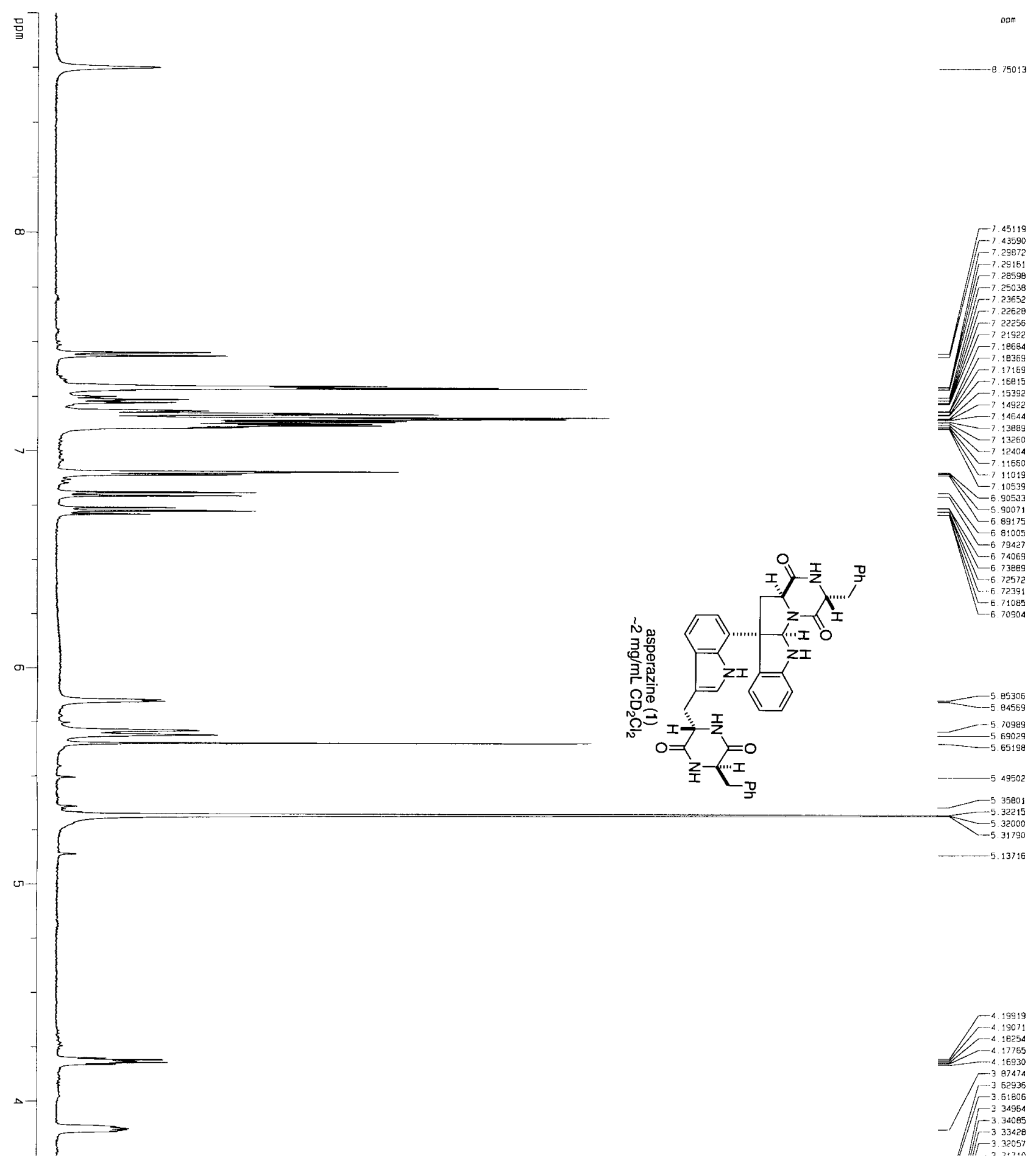




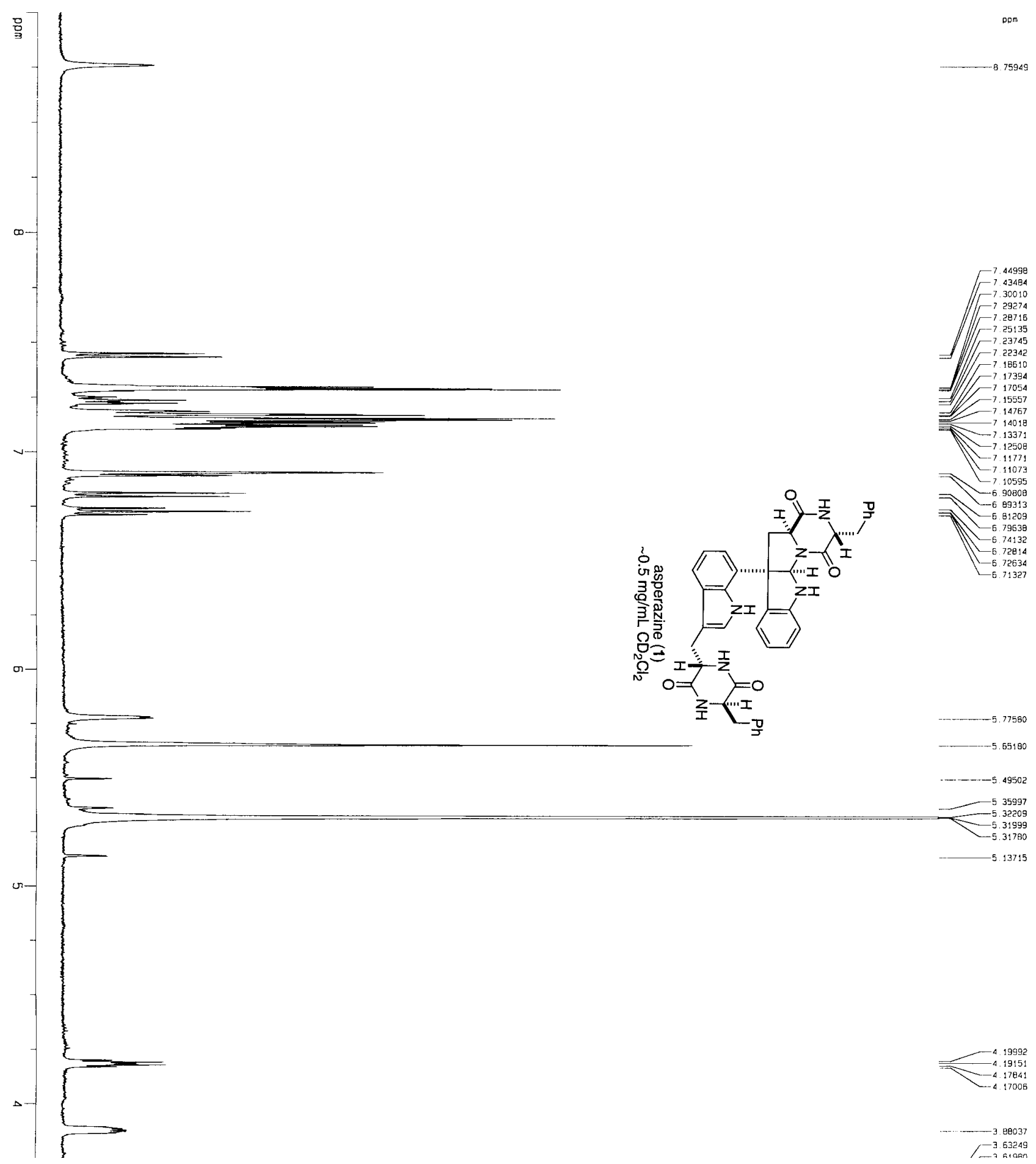



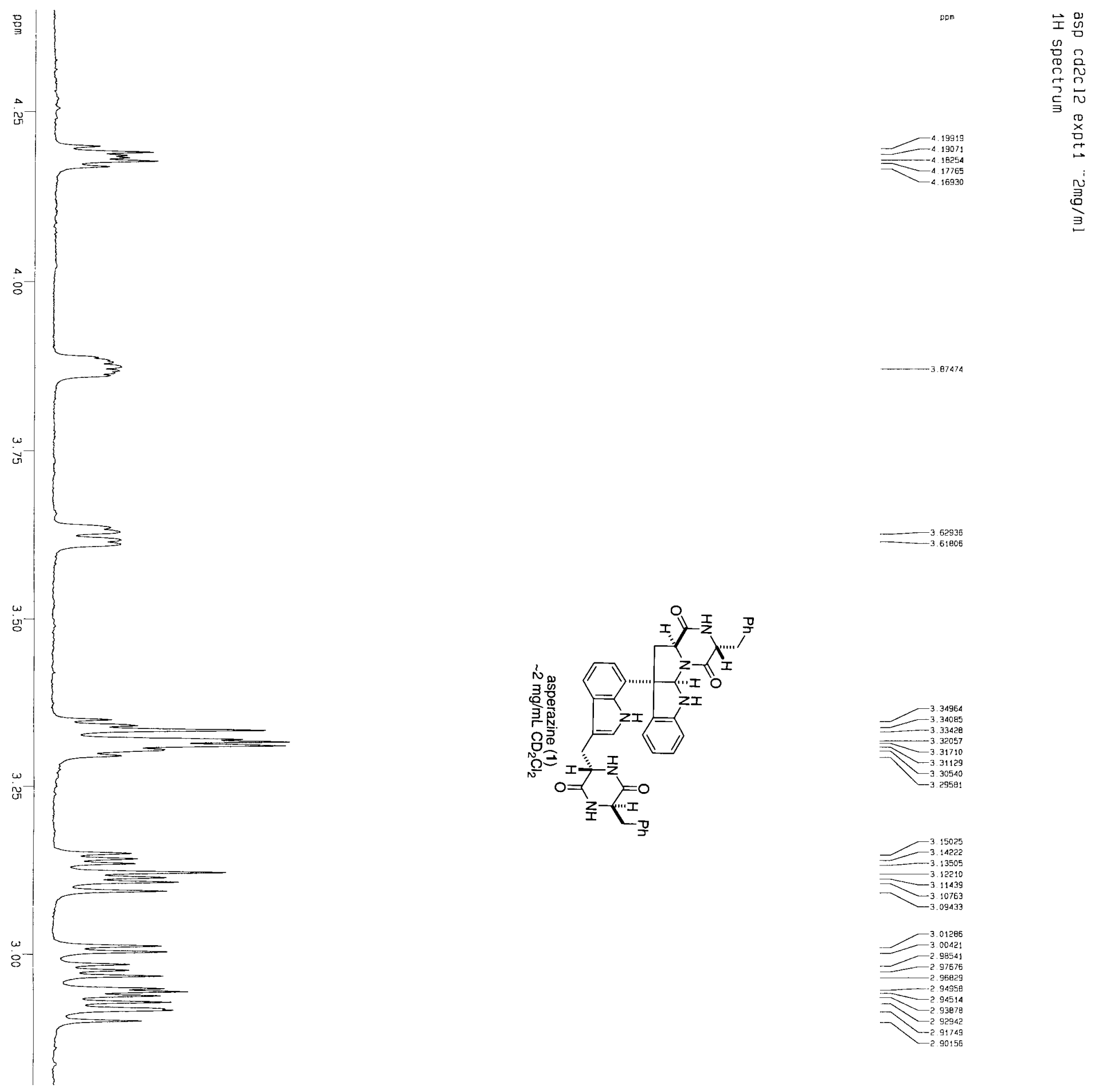


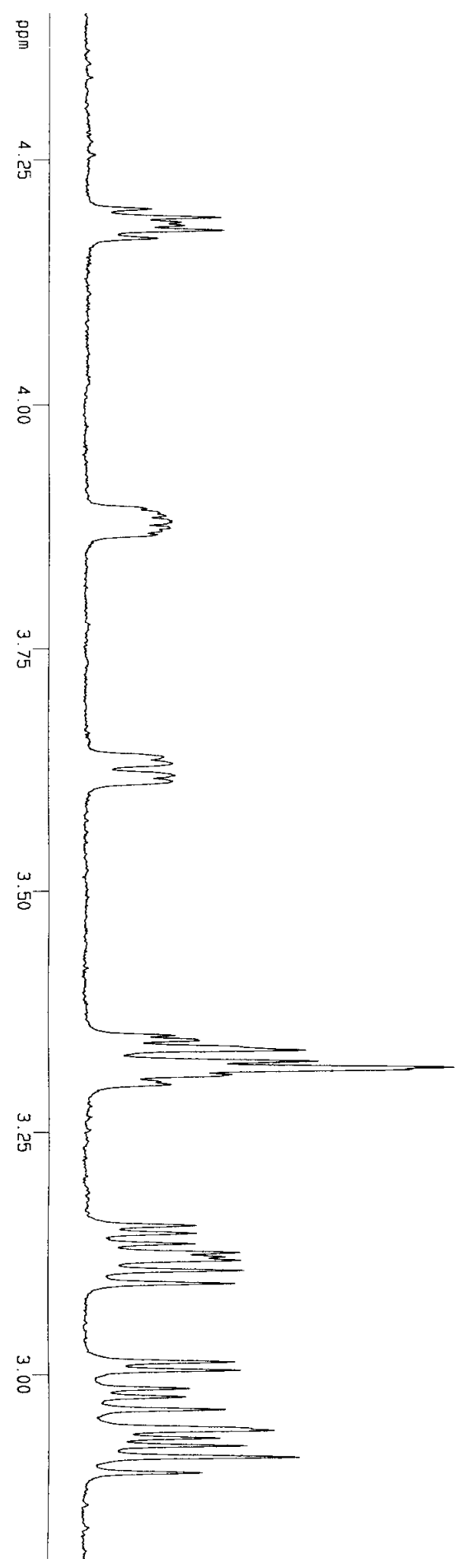

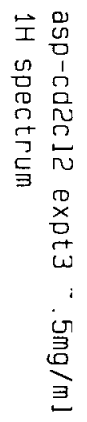
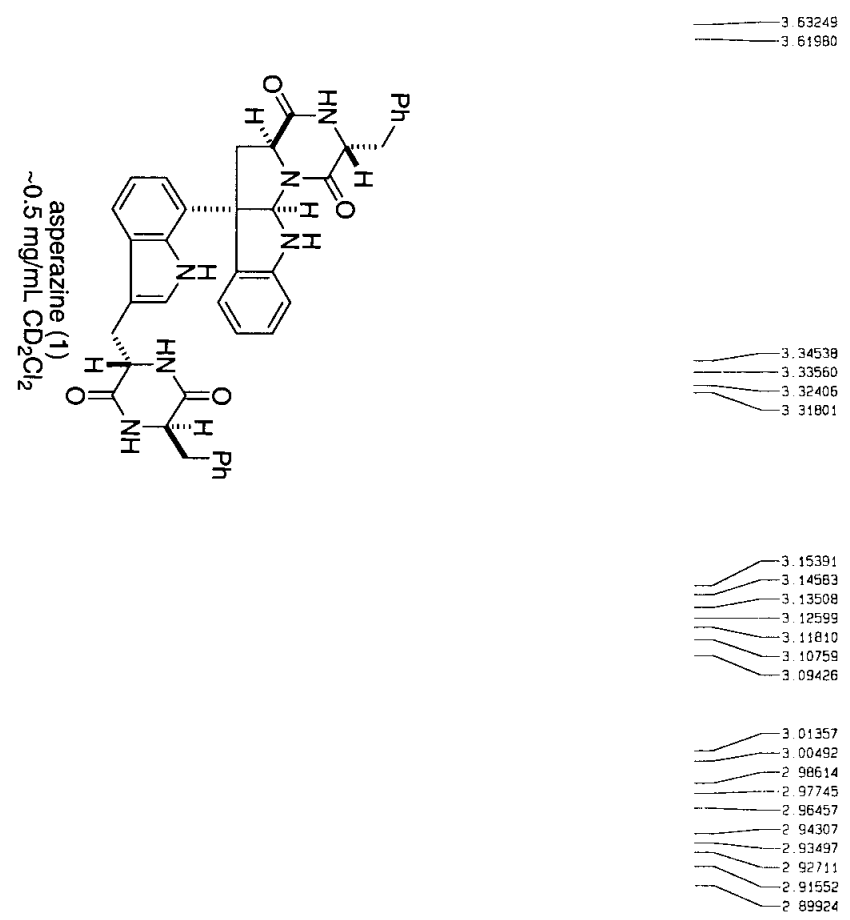


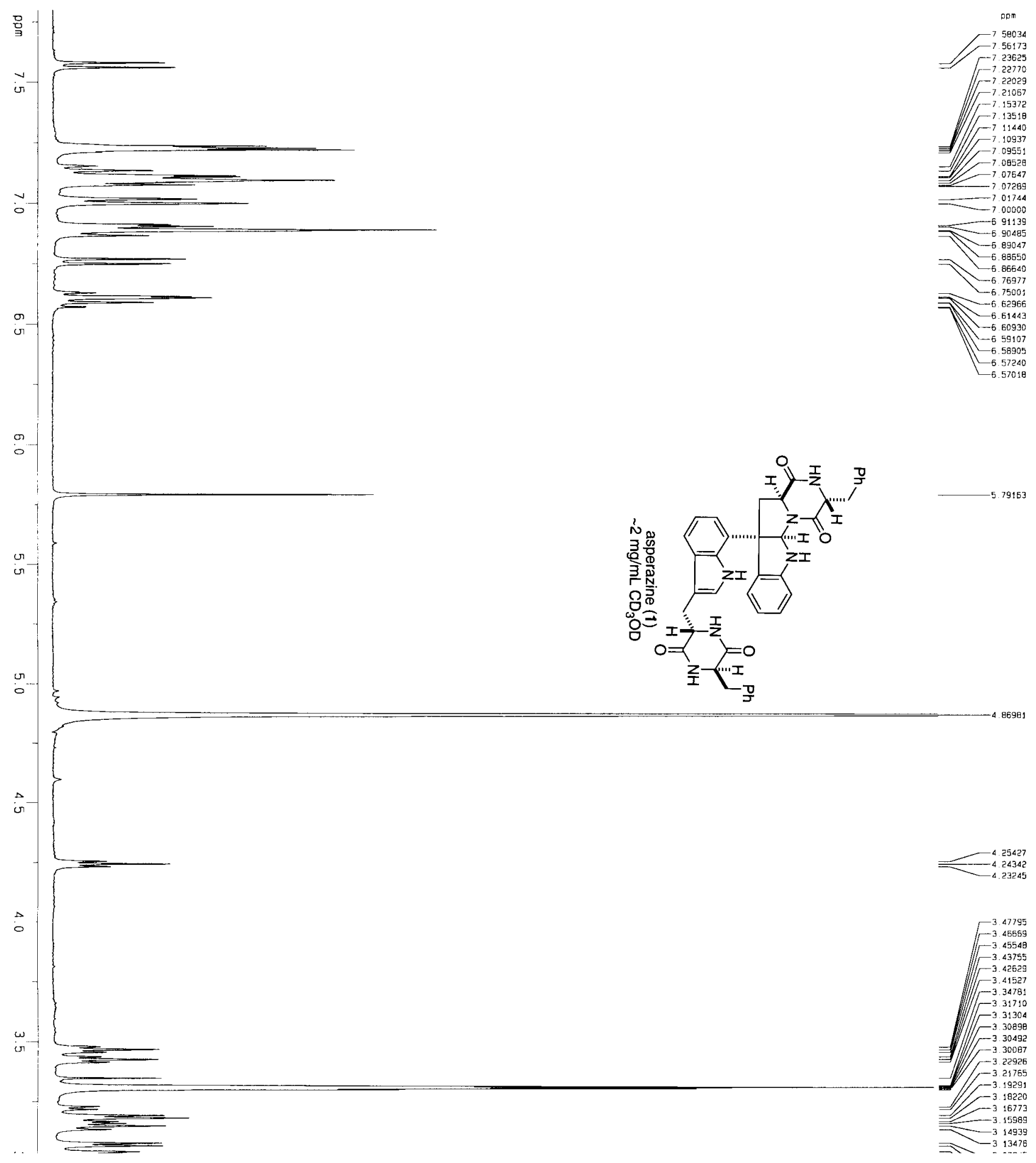



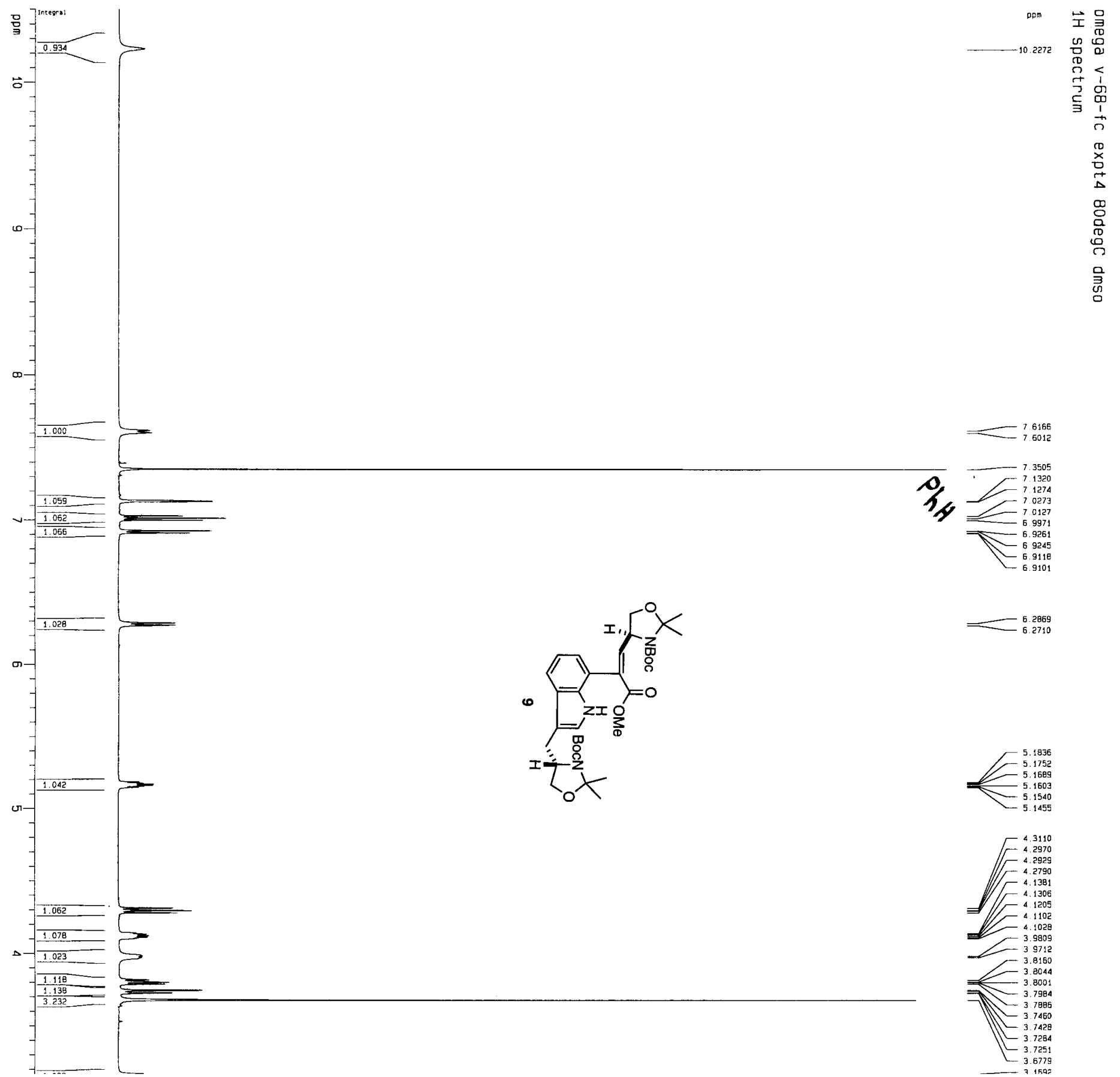

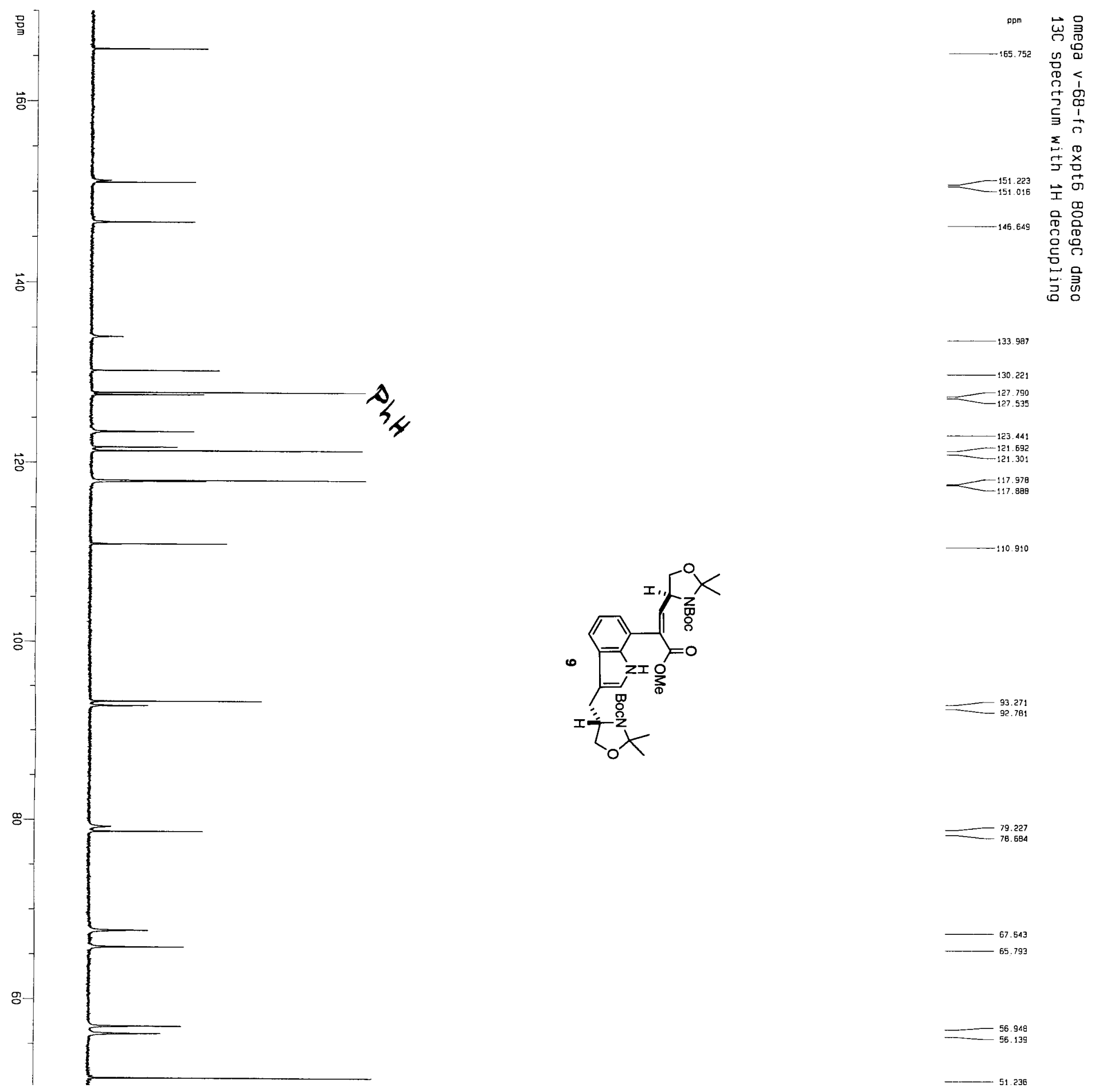


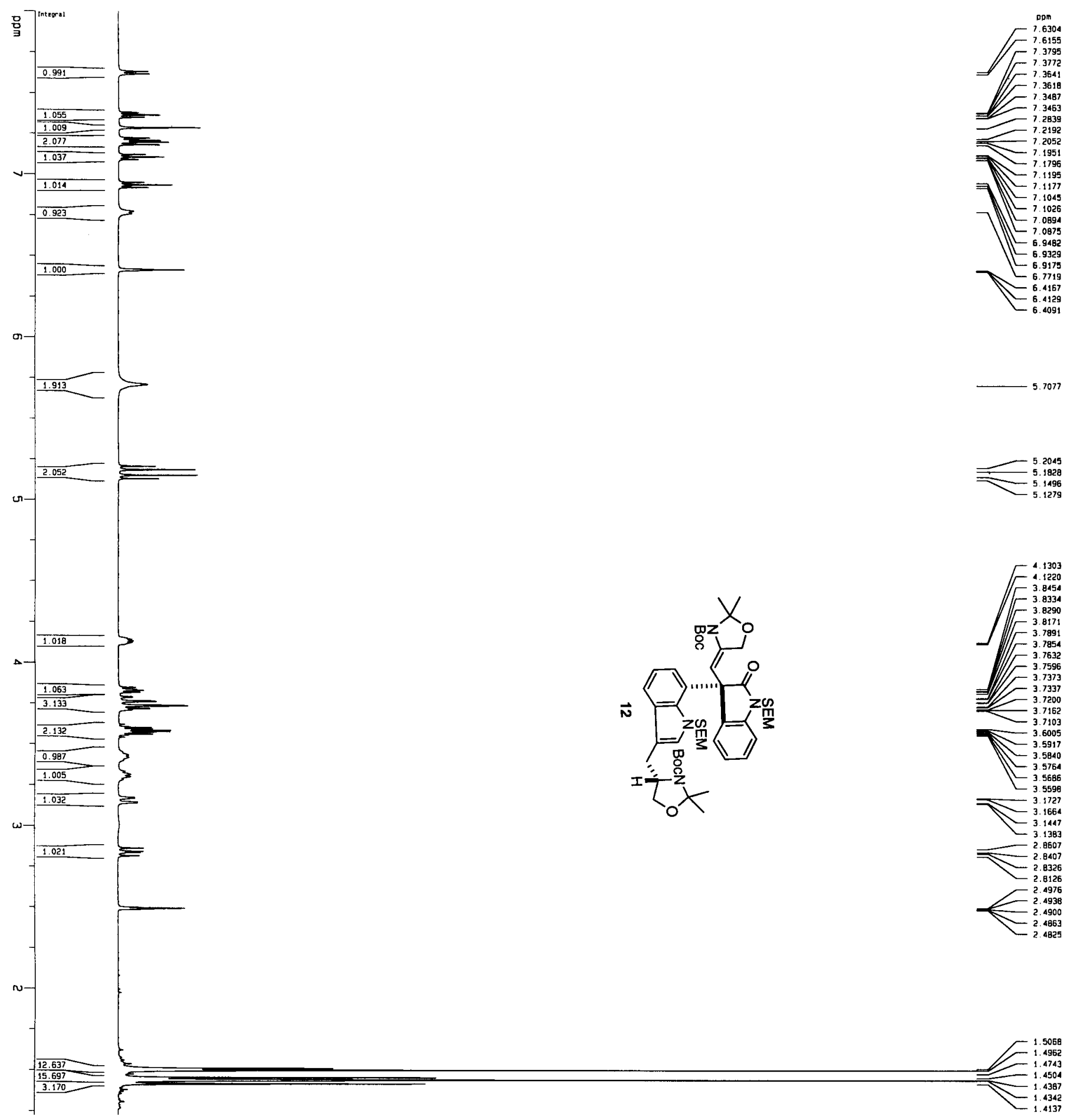



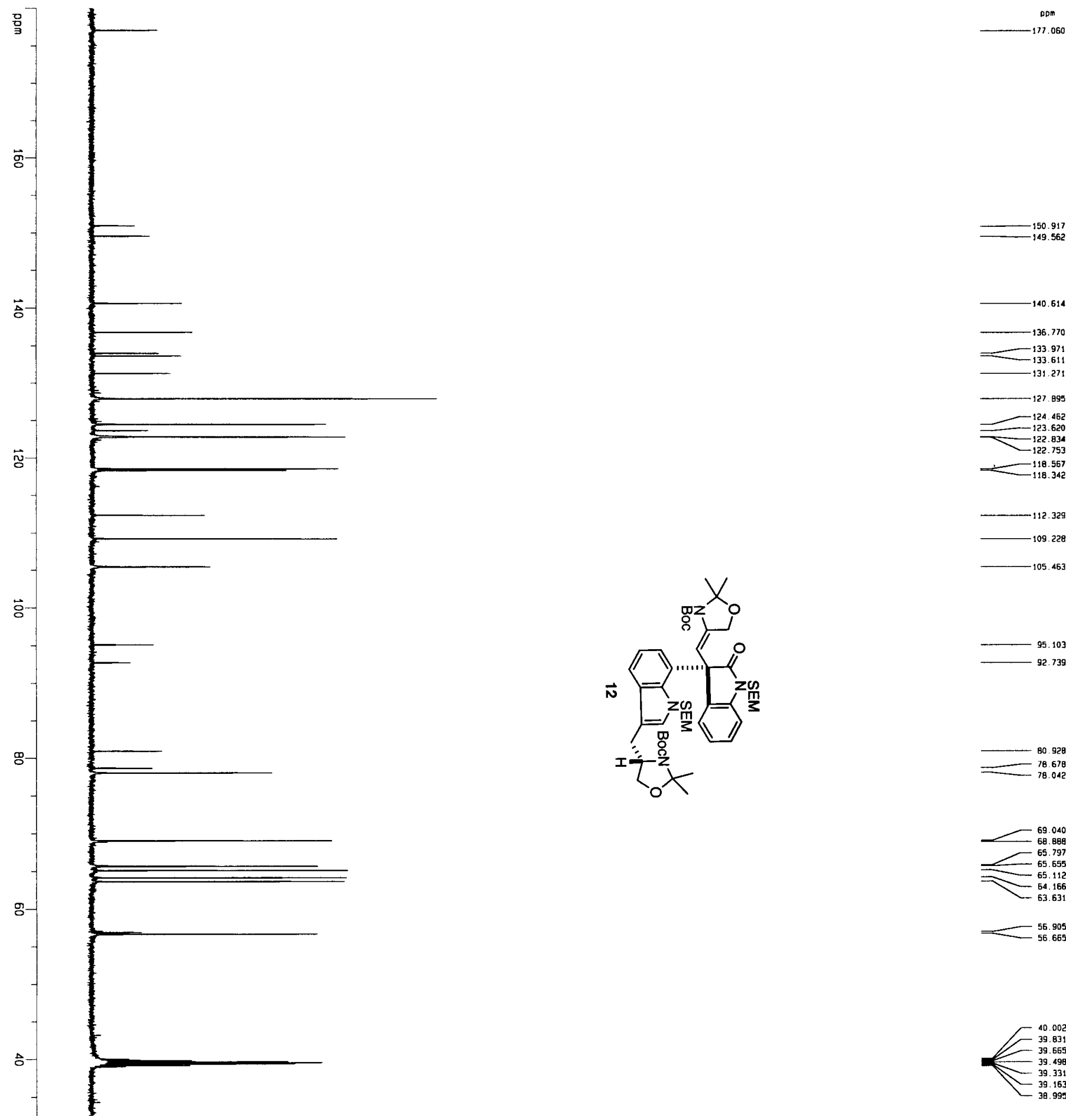

$-131.271$
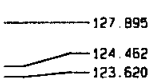
124.462
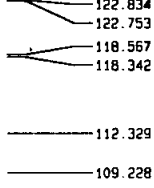

105. 228
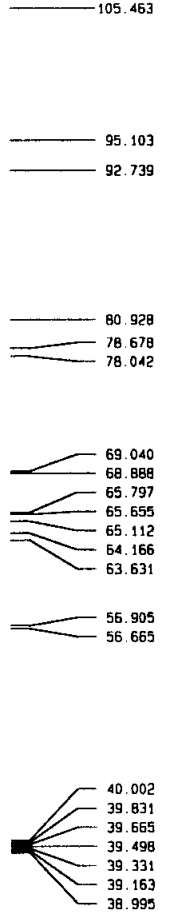

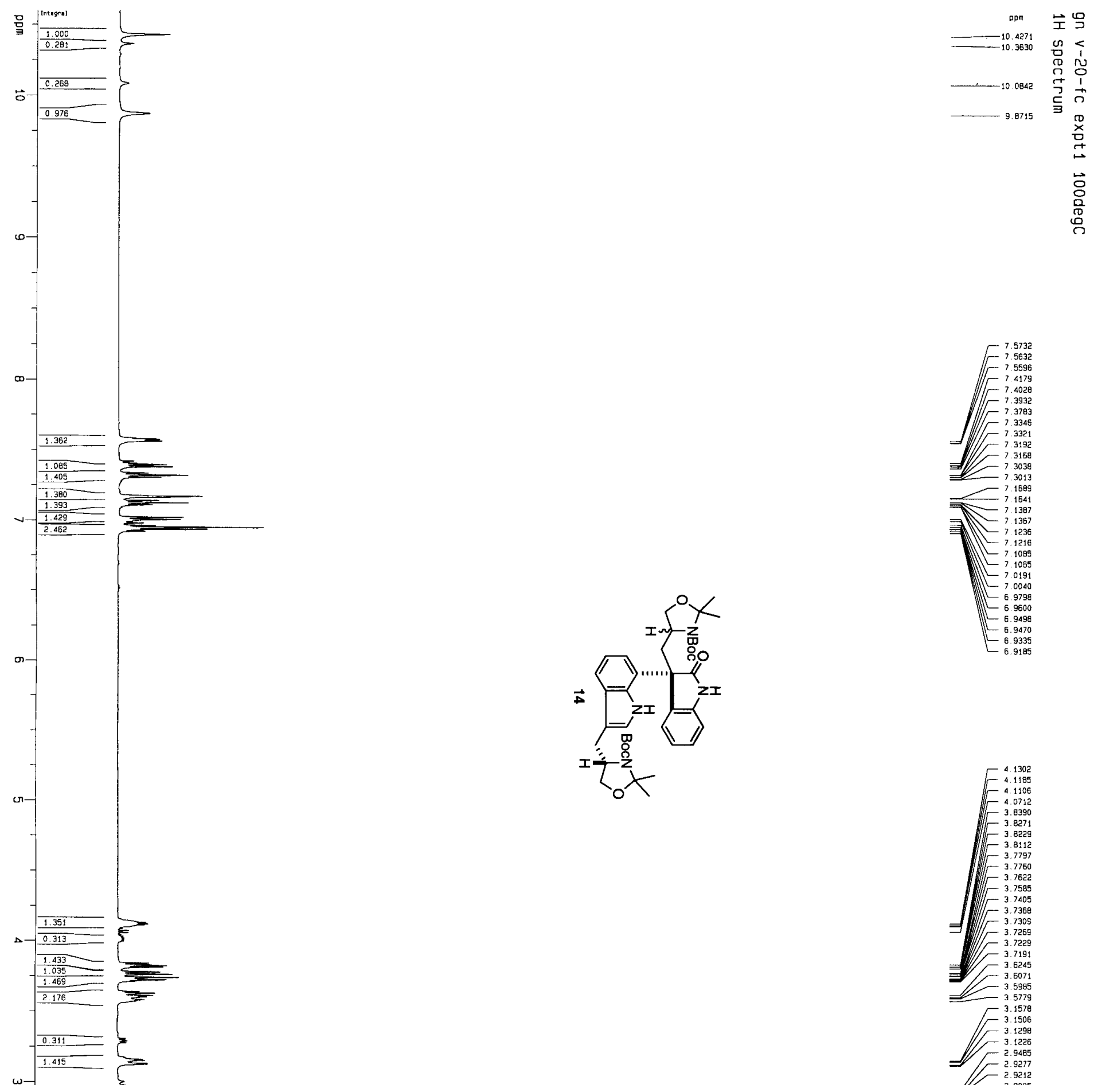

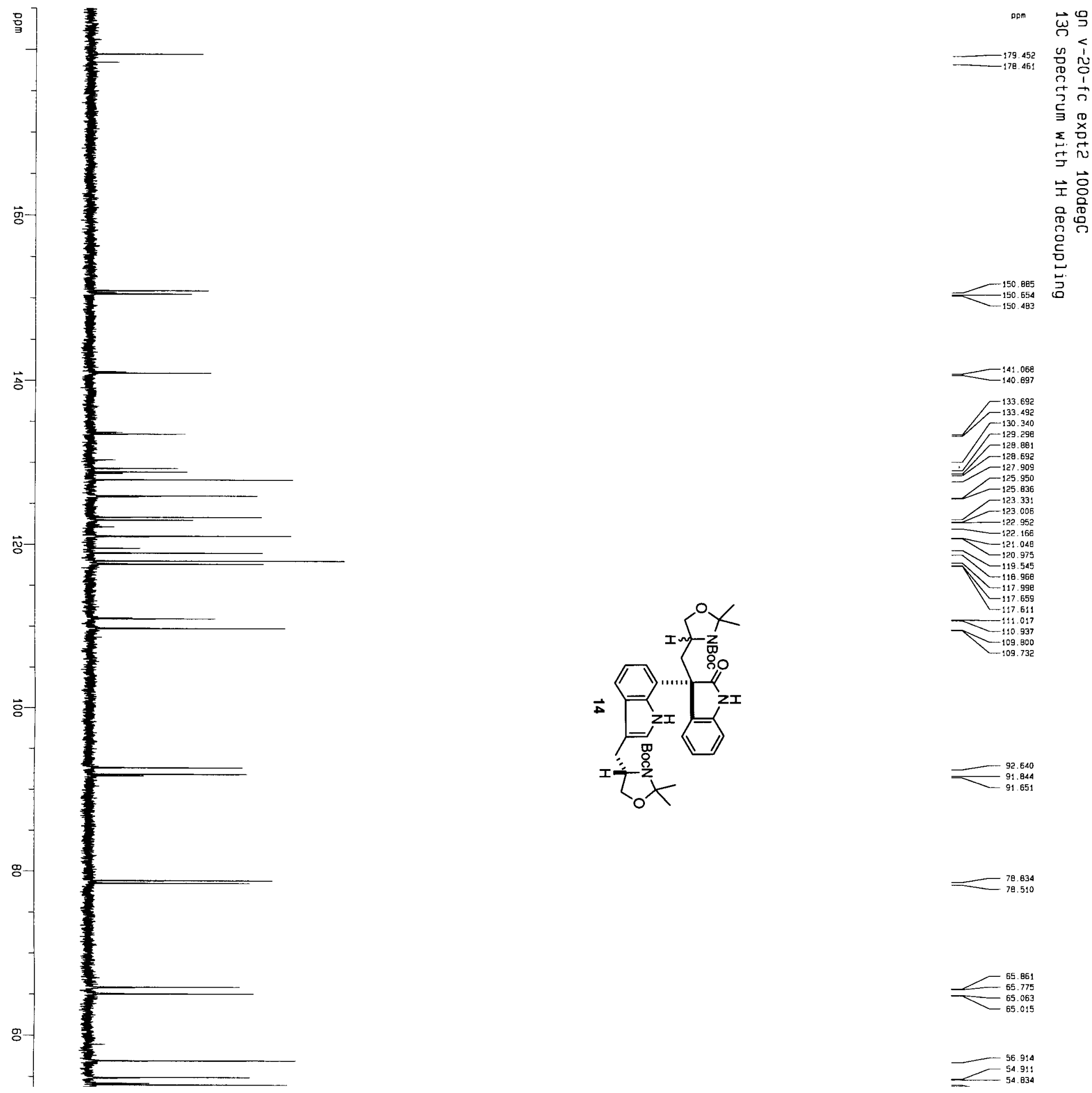